\title{
Stochastic Characteristics and Optimal Control for a Stochastic Chemostat Model with Variable Yield
}

\author{
Rong Yan and Shulin Sun \\ School of Mathematics and Computer Science, Shanxi Normal University, Linfen 041004, China \\ Correspondence should be addressed to Shulin Sun; sunshulin2013@163.com
}

Received 19 December 2019; Revised 4 March 2020; Accepted 2 April 2020; Published 28 April 2020

Academic Editor: Eulalia Martínez

Copyright ( 92020 Rong Yan and Shulin Sun. This is an open access article distributed under the Creative Commons Attribution License, which permits unrestricted use, distribution, and reproduction in any medium, provided the original work is properly cited.

In this paper, a stochastic chemostat model with variable yield and Contois growth function is investigated. The yield coefficient depends on the limiting nutrient, and the environmental noises are given by independent standard Brownian motions. First, the existence and uniqueness of global positive solution are proved. Second, by using stochastic Lyapunov function, Itô's formula, and some important inequalities, stochastic characteristics for the stochastic model are studied, including the extinction of microorganism, the strong persistence in the mean of micro-organism and, the existence of a unique stationary distribution of the stochastic model. Third, the necessary condition of an optimal stochastic control for the stochastic model is established by Hamiltonian function. In addition, some numerical simulations are carried out to illustrate the theoretical results and the influence of the variable yield on the microorganism

\section{Introduction}

The chemostat occupies a central place in mathematical ecology. It has been widely used in modeling natural ecosystems such as lakes and establishing waste-water treatment mathematical model. Many works related to the chemostat model have been published in the journal of mathematical, biological, and chemical engineering (see [1-5], etc.).

A large number of scholars are attracted to yield coefficient reflecting the conversion of nutrient to microorganism. Most of the models assume that the yield coefficient is a constant (see [1, 2]). Smith and Waltman [1] have described a chemostat model with constant yield as follows:

$$
\left\{\begin{array}{l}
S^{\prime}(t)=\left(S^{0}-S(t)\right) D-p(S, x) \frac{x(t)}{\gamma}, \\
x^{\prime}(t)=x(t)(p(S, x)-D),
\end{array}\right.
$$

where all the parameters are positive constants. $S(t)$ and $x(t)$ stand for the concentrations of the nutrient and the microorganism at time $t$ in culture vessel, respectively. $S^{0}$ is the input nutrient concentration. $D$ is the common dilution rate. $\gamma$ is a "yield" constant. $p(S, x)$ represents the growth function. Sun and Yin [2] and Wang et al. [3] have studied the chemostat model with Monod growth function. Bayen et al. [4] and Sun and Chen [5] have investigated the chemostat model with Contois growth function. Based on model (1), choosing Contois growth function $p(S, x)=(m S(t) / k x(t)+S(t))$, the model takes the following form:

$$
\left\{\begin{array}{l}
S^{\prime}(t)=\left(S^{0}-S(t)\right) D-\frac{m S(t)}{k x(t)+S(t)} \frac{x(t)}{\gamma}, \\
x^{\prime}(t)=x(t)\left(\frac{m S(t)}{k x(t)+S(t)}-D\right),
\end{array}\right.
$$

where $m>0$ represents the maximal growth rate and $k>0$ stands for the growth coefficient of the Contois function.

However, experimental data indicate that a constant yield may fail to explain the observed oscillatory behavior in the vessel (see [6]). This leads to the formulation of the variable yield model, for example [7-9]. The chemostat model with variable yield takes the following form: 


$$
\left\{\begin{array}{l}
S^{\prime}(t)=\left(S^{0}-S(t)\right) D-\frac{m S(t)}{k x(t)+S(t)} \frac{x(t)}{A+B S(t)} \\
x^{\prime}(t)=x(t)\left(\frac{m S(t)}{k x(t)+S(t)}-D\right),
\end{array}\right.
$$

where $\gamma(S)=A+B S$ is the variable yield which is considered in [9]. Both $A$ and $B$ are positive constants.

Actually, the above models are all deterministic models, but almost all ecosystems are inevitably perturbed by various types of environmental noises. Taking into account the realistic and biological significance, many scholars have investigated stochastic chemostat models (see [3, 10-14]) and stochastic population models (see [15-19]). In the chemostat model, the micro-organism may be affected by distribution of nutrient, temperature, humidity, etc, which can be described by continuous white noise. There are different possible approaches to include random effects in the model, both from a biological and mathematical perspective. Some authors have superimposed white noise processes on the dilution rate [20]; others have considered the linear white noise $[11,21]$. Our basic approach is analogous to that of Beddington and May [21]. By this method, the white noise are directly proportional to $S(t)$ and $x(t)$, influenced on $S^{\prime}(t)$ and $x^{\prime}(t)$. A stochastic chemostat model with variable yield and Contois growth function will be investigated, and the model (3) will be rewritten as the form:

$$
\left\{\begin{array}{l}
\mathrm{d} S(t)=\left(\left(S^{0}-S(t)\right) D-\frac{m S(t)}{k x(t)+S(t)} \frac{x(t)}{A+B S(t)}\right) \mathrm{d} t \\
+\sigma_{1} S(t) \mathrm{d} B_{1}(t) \\
\mathrm{d} x(t)=x(t)\left(\frac{m S(t)}{k x(t)+S(t)}-D\right) \mathrm{d} t+\sigma_{2} x(t) \mathrm{d} B_{2}(t),
\end{array}\right.
$$

where $\sigma_{i}>0(i=1,2)$ are intensities of the white noise. $B_{i}(t)(i=1,2)$ are independent standard Brownian motions defined on a complete probability space $\left(\Omega, \mathscr{F},\left\{\mathscr{F}_{t}\right\}_{t \geq 0}, \mathbb{P}\right)$ with the filtration $\left\{\mathscr{F}_{t}\right\}_{t \geq 0}$ satisfying the usual conditions. The model (4) turns into the corresponding deterministic model (3) if the noise intensities $\sigma_{i}=0(i=1,2)$.

The initial conditions of (4) are given as

$$
\begin{aligned}
& S(0)=S_{0}, \\
& x(0)=x_{0},
\end{aligned}
$$

where $S_{0}, x_{0}$ are positive random variables, and denote $\mathbb{R}_{+}^{2}=\left\{\left(x_{1}, x_{2}\right) \mid x_{i}>0, i=1,2\right\}$.

Because the solutions of the deterministic model (3) are no longer the solutions of the stochastic model (4), the dynamic behavior of the deterministic model (3) is distinct from that of the stochastic model (4). For further details on the stochastic model, please see [10-14], they have investigated the stochastic characteristics of these stochastic models. However, the nonlinear term of the model (4) is different from that of their models. Therefore, it is valuable to investigate the stochastic characteristics of the stochastic chemostat model with variable yield and Contois growth function. On the other hand, the optimal control plays an important role in practical application $[4,22]$. Many scholars have paid more attention to the optimal stochastic control problem, which covers all aspects of physics, biology, economics, etc. Ding et al. [23] have solved the distributed $H_{\infty}$ state estimation problem for a class of discrete time-varying nonlinear system with both stochastic parameters and stochastic nonlinearities. Guo et al. [24] have studied the near-optimal control of a stochastic SIRS epidemic model that includes a nonmonotone incidence rate. Framstad et al. [25] have proved a sufficient maximum principle for the optimal control of jump diffusions and showed its connections to dynamic programming and given applications to financial optimization problems in a market described by such processes. Thus, it is necessary to study the optimal stochastic control of a stochastic chemostat model. Throughout the whole paper, Lyapunov function, Itô formula, and other basic methods can be referred to these monographs [26-32].

This paper is organized as follows. In Section 2, the existence of the unique global positive solution for the model (4) is studied. In Section 3, the stochastic characteristics of the stochastic model (4) are investigated, including the extinction at an exponential rate of the microorganism, the strong persistence in the mean of the microorganism, and the stationary distribution of the model (4). In Section 4, the necessary condition of an optimal stochastic control for the stochastic model (4) is investigated, and the near-optimal stochastic control is mentioned. In Section 5, the numerical simulations conclude the paper, and the influence of the variable yield on the microorganism is explained by taking the different parameters.

\section{Preliminaries}

First of all, the notations are described for the whole paper as follows:

(i) $\Omega$ : a set of the elementary events

(ii) $\mathscr{F}$ : a family of the subsets of $\Omega$

(iii) $\left\{\mathscr{F}_{t}\right\}_{t \geq 0}$ : a family of increasing sub- $\sigma$-algebras of $\mathscr{F}$

(iv) $\mathbb{P}(\omega)$ : the probability of events $\omega$

(v) $\mathbb{E} X$ : the expectation of $X$

(vi) $\varnothing$ : the empty set

(vii) $I_{A}$ : the indicator function of a set $A$, i.e., $I_{A}(x)=1$ if $x \in A$ or otherwise 0

(viii) a.s.: almost surely

(ix) $E^{n}$ : the $n$-dimensional Euclidean space

(x) $\mathbb{R}$ : the set of all real numbers

(xi) $\mathbb{R}_{+}$: the set of all nonnegative real numbers, i.e., $\mathbb{R}_{+}=[0, \infty)$

(xii) $\mathbb{R}_{+}^{d}:\left\{x \in \mathbb{R}^{d}: x_{i}>0,1 \leq i \leq d\right\}$, i.e. the positive cone 
(xiii) $U$ : an open domain

(xiv) $\bar{U}$ : a close domain

In view of biological significance and dynamical behavior, the first concerned thing is whether the solution is unique, global, and positive. Hence, to further study the stochastic chemostat model with variable yield and Contois growth function (4), the first problem to be solved is the existence of the unique global positive solution, namely, there is no explosion in a finite time under the initial value (5). If the coefficients of the equations are generally required to satisfy the linear growth condition and the local Lipschitz condition (see $[28,30])$, the stochastic differential equations for any given initial value have a unique global solution. However, the stochastic model (4) may allow the solutions to explode at a finite time because the coefficients of the stochastic model (4) do not satisfy the linear growth condition. So, we need to search for the positive solutions. The following theorem assures that the solution of the model (4) with the initial value (5) is unique, global, and positive. First, a lemma and a remark are given.

Lemma 1 (see [19]). The following inequality holds:

$$
u \leq 2(u+1-\ln u)-(4-2 \ln 2), \forall u>0 .
$$

Remark 1. Based on Lemma 1 , since the inequality $u \leq 2(u+$ $1-\ln u)-(4-2 \ln 2)$, we can obtain $u \leq 2(u+1-\ln u)$ holds for all $u>0$.

Next, the existence of the unique global positive solution for the model (4) is proved.

Theorem 1. For any initial value $\left(S_{0}, x_{0}\right) \in \mathbb{R}_{+}^{2}$, there exists a unique positive solution $(S(t), x(t))$ to the model (4) for $t \geq 0$, and the solution will remain in $\mathbb{R}_{+}^{2}$ with probability one (i.e., $(S(t), x(t)) \in \mathbb{R}_{+}^{2}$ for all $t \geq 0$ a.s. $)$.

Proof. Since the coefficients of the stochastic differential equations (4) satisfy the local Lipschitz condition, the model (4) has a unique positive local solution $(S(t), x(t))$ on $t \in\left[0, \tau_{e}\right)$ for any initial value $\left(S_{0}, x_{0}\right) \in \mathbb{R}_{+}^{2}$, where $\tau_{e}$ is the explosion time. In order to show the solution is global, we just need to prove that $\tau_{e}=\infty$ a.s.. Select $k_{0} \geq 1$ sufficiently large such that $S_{0}$ and $x_{0}$ all lie within the interval $\left[\left(1 / k_{0}\right), k_{0}\right]$. For $\forall k \geq k_{0}$, where $k$ is a integer, define the stopping time

$$
\tau_{k}=\inf \left\{t \in\left[0, \tau_{e}\right) \mid S(t) \notin\left(\frac{1}{k}, k\right) \text { or } x(t) \notin\left(\frac{1}{k}, k\right)\right\} .
$$

Throughout this paper, we set inf $\varnothing=\infty$. $\tau_{k}$ is increasing when $k \longrightarrow \infty$. Let $\tau_{\infty}=\lim _{k \longrightarrow+\infty} \tau_{k}$, clearly, $\tau_{\infty} \leq \tau_{e}$ a.s. $\tau_{e}=\infty$ and $(S(t), x(t)) \in \mathbb{R}_{+}^{2}$ a.s. for all $t \geq 0$ if $\tau_{\infty}=\infty$ can be verified. Next, for $\tau_{\infty}=\infty$, the proof process is as follows:

Define a $C^{2}$-function $V: \mathbb{R}_{+}^{2} \longrightarrow \mathbb{R}_{+}$by

$$
V(S, x)=S+1-\ln S+x+1-\ln x,
$$

for $\forall T>0$, by applying Itô's formula on $t \in\left[0, \tau_{k} \wedge T\right]$, we have

$$
\begin{aligned}
\mathrm{d} V(S, x)= & \left(1-\frac{1}{S}\right) \mathrm{d} S+\left(1-\frac{1}{x}\right) \mathrm{d} x+\frac{1}{2} \frac{1}{S^{2}}(\mathrm{~d} S)^{2}+\frac{1}{2} \frac{1}{x^{2}}(\mathrm{~d} x)^{2} \\
= & \left(\left(1-\frac{1}{S}\right)\left(S^{0}-S\right) D-(S-1) \frac{m x}{(k x+S)(A+B S)}\right. \\
& \left.+(x-1)\left(\frac{m S}{k x+S}-D\right)+\frac{1}{2} \sigma_{1}^{2}+\frac{1}{2} \sigma_{2}^{2}\right) \mathrm{d} t+\sigma_{1} \\
& \cdot(S-1) \mathrm{d} B_{1}(t)+\sigma_{2}(x-1) \mathrm{d} B_{2}(t) \\
= & L V \mathrm{~d} t+\sigma_{1}(S-1) \mathrm{d} B_{1}(t)+\sigma_{2}(x-1) \mathrm{d} B_{2}(t),
\end{aligned}
$$

by using Lemma 1

$$
\begin{aligned}
L V= & S^{0} D-S D-\frac{S^{0}}{S} D+D-\frac{m S x}{(k x+S)(A+B S)} \\
& +\frac{m x}{(k x+S)(A+B S)}+\frac{m S x}{k x+S} \\
& -x D-\frac{m S}{k x+S}+D+\frac{1}{2} \sigma_{1}^{2}+\frac{1}{2} \sigma_{2}^{2} \\
\leq & S^{0} D+2 D+\frac{1}{2} \sigma_{1}^{2}+\frac{1}{2} \sigma_{2}^{2}+\frac{m x}{(k x+S)(A+B S)}+\frac{m S x}{k x+S} \\
\leq & S^{0} D+2 D+\frac{1}{2} \sigma_{1}^{2}+\frac{1}{2} \sigma_{2}^{2}+\frac{m}{k A}+m x \\
\leq & S^{0} D+2 D+\frac{1}{2} \sigma_{1}^{2}+\frac{1}{2} \sigma_{2}^{2}+\frac{m}{k A}+2 m(x+1-\ln x) \\
& +2(S+1-\ln S) \\
\triangleq & N_{1}+N_{2} V(S, x),
\end{aligned}
$$

so,

$$
\begin{aligned}
\mathrm{d} V(S, x) \leq & \left(N_{1}+N_{2} V(S, x)\right) \mathrm{d} t+\sigma_{1}(S-1) \mathrm{d} B_{1}(t) \\
& +\sigma_{2}(x-1) \mathrm{d} B_{2}(t),
\end{aligned}
$$

where

$$
\begin{aligned}
& N_{1}=S^{0} D+2 D+\frac{1}{2} \sigma_{1}^{2}+\frac{1}{2} \sigma_{2}^{2}+\frac{m}{k A}, \\
& N_{2}=2 m \vee 2 .
\end{aligned}
$$

Integrating equation (11) both sides from 0 to $\tau_{k} \wedge T$ and taking expectation,

$$
\begin{aligned}
\mathbb{E} V\left(S\left(\tau_{k} \wedge T\right), x\left(\tau_{k} \wedge T\right)\right) \leq & V\left(S_{0}, x_{0}\right)+N_{1} T+N_{2} \mathbb{E} \\
& \cdot \int_{0}^{\tau_{k} \wedge T} V(S(t), x(t)) \mathrm{d} t .
\end{aligned}
$$

By using Gronwall's inequality, we have

$\mathbb{E} V\left(S\left(\tau_{k} \wedge T\right), x\left(\tau_{k} \wedge T\right)\right) \leq\left(V\left(S_{0}, x_{0}\right)+N_{1} T\right) e^{N_{2} T}$. 
For $\forall \omega \in\left\{\tau_{k} \leq T\right\}$, it exists that $S\left(\tau_{k}, \omega\right)$ or $x\left(\tau_{k}, \omega\right)$ equals either $k$ or $(1 / k)$,

$$
\begin{aligned}
V\left(S\left(\tau_{k}, \omega\right), x\left(\tau_{k}, \omega\right)\right) & \geq(k+1-\ln k) \wedge\left(\frac{1}{k}+1+\ln k\right) \\
& \triangleq G(k) .
\end{aligned}
$$

Thus,

$$
\begin{aligned}
\left(V\left(S_{0}, x_{0}\right)+N_{1} T\right) e^{N_{2} T} & \geq \mathbb{E}\left[I_{\left\{\tau_{k} \leq T\right\}}(\omega) V\left(S\left(\tau_{k}, \omega\right), x\left(\tau_{k}, \omega\right)\right)\right] \\
& \geq \mathbb{P}\left\{\tau_{k} \leq T\right\} G(k),
\end{aligned}
$$

where $I_{\left\{\tau_{k} \leq T\right\}}$ is the indicator function of $\left\{\tau_{k} \leq T\right\}$.

Letting $k \longrightarrow \infty$ gives $\lim _{k \longrightarrow \infty} \mathbb{P}\left\{\tau_{k} \leq T\right\}=0$, and hence, $\mathbb{P}\left\{\tau_{\infty} \leq T\right\}=0$. Since $T>0$ is arbitrary, we must have

$$
\mathbb{P}\left\{\tau_{\infty}<\infty\right\}=0
$$

so $\mathbb{P}\left\{\tau_{\infty}=\infty\right\}=1$ as required.

The proof is thus complete.

\section{Stochastic Characteristics}

In this section, the stochastic characteristics of the stochastic model (4) are discussed. As is known to all, the equilibriums of the deterministic model (3) are no longer the equilibriums of the stochastic model (4), then what happens in the stochastic model (4)? Next, the sufficient conditions of the extinction and persistence of microorganism and the stationary distribution of the model (4) are investigated.

In order to study the model (4), we introduce the following definitions and lemmas, and denote $\langle x\rangle=(1 / t) \int_{0}^{t} x(s) \mathrm{d} s$.

Lemma 2 (see [14]). For any initial value (5), the solutions $S(t)$ and $x(t)$ of the model (4) have the properties that

$$
\begin{aligned}
& \limsup _{t \longrightarrow \infty} S(t)<\infty, \\
& \limsup _{t \longrightarrow \infty} x(t)<\infty,
\end{aligned}
$$

$$
\text { a.s.. }
$$
that

That is, there are two positive constants $H_{1}$ and $H_{2}$ such

$$
\begin{gathered}
\qquad(t) \leq H_{1}, \\
x(t) \leq H_{2}, \\
\text { for all } t \geq 0, \\
\text { a.s.. }
\end{gathered}
$$

Lemma 3 (see [28]). Let $M=\left\{M_{t}\right\}_{t \geq 0}$ be a real-valued continuous local martingale vanishing at $t=0$. Then

$$
\begin{aligned}
\limsup _{t \rightarrow \infty} \frac{\langle M, M\rangle_{t}}{t} & <\infty, \quad \text { a.s. } \\
& \Rightarrow \lim _{t \rightarrow \infty} \frac{M_{t}}{t}=0, \quad \text { a.s.. }
\end{aligned}
$$

Next, the extinction at an exponential rate of the microorganism is studied.

Theorem 2. If the condition holds,

$$
\sigma_{2}^{2}>2(m-D)
$$

then for any initial value (5), the solution $(S(t), x(t))$ of the model (4) satisfies

$$
\underset{t \rightarrow \infty}{\limsup } \frac{\ln x(t)}{t} \leq m-D-\frac{1}{2} \sigma_{2}^{2}<0, \quad \text { a.s. }
$$

which means

$$
\lim _{t \rightarrow \infty} x(t)=0, \quad \text { a.s.. }
$$

That is, the microorganism will be extinct at an exponential rate with probability one.

Proof. Define a function

$$
V=\ln x
$$

using Itô formula

$$
\begin{aligned}
\mathrm{d} V & =\left(\frac{m S}{k x+S}-D-\frac{1}{2} \sigma_{2}^{2}\right) \mathrm{d} t+\sigma_{2} \mathrm{~d} B_{2}(t) \\
& \leq\left(m-D-\frac{1}{2} \sigma_{2}^{2}\right) \mathrm{d} t+\sigma_{2} \mathrm{~d} B_{2}(t), \quad \text { a.s.. }
\end{aligned}
$$

Integrating both sides from 0 to $t$, we can obtain

$$
\frac{V(t)}{t}-\frac{V(0)}{t} \leq m-D-\frac{1}{2} \sigma_{2}^{2}+\frac{\sigma_{2}}{t} B_{2}(t),
$$

then

$$
\frac{\ln x(t)}{t} \leq m-D-\frac{1}{2} \sigma_{2}^{2}+\varphi_{1}(t), \quad \text { a.s. }
$$

where

$$
\begin{aligned}
\varphi_{1}(t) & =\frac{\sigma_{2}}{t} B_{2}(t)+\frac{\ln x_{0}}{t}, \\
\lim _{t \rightarrow \infty} \varphi_{1}(t) & =0,
\end{aligned}
$$

$$
\text { a.s.. }
$$

According to equation (27), we have

$$
\underset{t \rightarrow \infty}{\limsup } \frac{\ln x(t)}{t} \leq m-D-\frac{1}{2} \sigma_{2}^{2}<0, \quad \text { a.s., }
$$

which implies that

$$
\lim _{t \rightarrow \infty} x(t)=0, \quad \text { a.s.. }
$$

Thus, the proof is completed. 
Remark 2. Theorem 2 shows that the condition $\sigma_{2}^{2}>2(m-$ $D)$ makes the microorganism extinct, but it has nothing to do with $m<D$ or $m>D$, which means that large noises can lead to the extinction of the microorganism, although the microorganism is persistent in the corresponding deterministic model (3) (see Figures 1 and 2)

A definition and a lemma are given before discussing the strong persistence in the mean of the microorganism.

Definition 1 (see [17]). The population $x(t)$ is said to be strong persistence in the mean if

$$
\langle x\rangle_{*}>0,
$$

where $\langle x\rangle_{*}=\liminf _{t \rightarrow \infty}\langle x\rangle$.

Lemma 4 (see [18]). Let $f \in C[[0, \infty) \times \Omega,(0, \infty)]$. If there exist positive constants $\lambda_{0}, \lambda$ such that

$$
\ln f(t) \geq \lambda t-\lambda_{0} \int_{0}^{t} f(s) \mathrm{d} s+F(t), \quad \text { a.s. }
$$

for all $t \geq 0$, where $F \in C[[0, \infty) \times \Omega, \mathbb{R}]$ and $\lim _{t \rightarrow \infty}(F(t) / t)=0$, a.s., then

$$
\langle f\rangle_{*} \geq \frac{\lambda}{\lambda_{0}}, \quad \text { a.s.. }
$$

Theorem 3. For any initial value (5), if the condition holds:

$$
\frac{1}{2} \sigma_{2}^{2}<\zeta \triangleq \min \left\{\frac{m}{k H_{2}+1}-D, \frac{m S^{0}}{k H_{2}+1}-D\right\},
$$

then the solution $(S(t), x(t))$ of the model (4) satisfies

$$
\langle x(t)\rangle_{*} \geq \frac{A D\left(k H_{2}+1\right)}{m(m+A \quad D)}\left(\zeta-\frac{1}{2} \sigma_{2}^{2}\right)>0 .
$$

That is, the microorganism is almost surely strongly persistent in the mean.

Proof. On the one hand, based on the model (4), we have

$$
\begin{aligned}
\mathrm{d}(S+x) & =\left(S^{0} D-S D-\frac{m S x}{(k x+S)(A+B S)}+\frac{m S x}{k x+S}-x D\right) \mathrm{d} t+\sigma_{1} S \mathrm{~d} B_{1}(t)+\sigma_{2} x \mathrm{~d} B_{2}(t) \\
& \geq\left(S^{0} D-S D-\frac{m x}{A}-x D\right) \mathrm{d} t+\sigma_{1} S \mathrm{~d} B_{1}(t)+\sigma_{2} x \mathrm{~d} B_{2}(t) \\
& =\left(S^{0} D-S D-\frac{m+A D}{A} x\right) \mathrm{d} t+\sigma_{1} S \mathrm{~d} B_{1}(t)+\sigma_{2} x \mathrm{~d} B_{2}(t),
\end{aligned}
$$

so,

$$
\begin{aligned}
\frac{S(t)+x(t)}{t}-\frac{S_{0}+x_{0}}{t} \geq & S 0-D\langle S(t)\rangle-\frac{m+A D}{A}\langle x(t)\rangle \\
& +\frac{\sigma_{1}}{t} M_{1}(t)+\frac{\sigma_{2}}{t} M_{2}(t),
\end{aligned}
$$

where

$$
\begin{aligned}
& M_{1}(t)=\int_{0}^{t} S(s) \mathrm{d} B_{1}(s), \\
& M_{2}(t)=\int_{0}^{t} x(s) \mathrm{d} B_{2}(s) .
\end{aligned}
$$

Thus,

$$
\langle S(t)\rangle \geq S^{0}-\frac{m+A \quad D}{A \quad D}\langle x(t)\rangle-\varphi_{2}(t),
$$

where

$$
\varphi_{2}(t)=\frac{1}{D}\left(\frac{S(t)+x(t)}{t}-\frac{S_{0}+x_{0}}{t}-\frac{\sigma_{1}}{t} M_{1}(t)-\frac{\sigma_{2}}{t} M_{2}(t)\right) .
$$

Since $M_{1}(t)$ and $M_{2}(t)$ are local continuous martingales with $M_{1}(0)=0$ and $M_{2}(0)=0$, from Theorem 5.14 in Mao's monograph [28], Lemmas 2 and 3, we have

$$
\begin{aligned}
& \limsup _{t \rightarrow \infty} \frac{\left\langle M_{1}, M_{1}\right\rangle_{t}}{t}=\frac{\int_{0}^{t} S^{2}(s) \mathrm{d} s}{t} \leq H_{1}^{2}<\infty, \\
& \limsup _{t \rightarrow \infty} \frac{\left\langle M_{2}, M_{2}\right\rangle_{t}}{t}=\frac{\int_{0}^{t} x^{2}(s) \mathrm{d} s}{t} \leq H_{2}^{2}<\infty,
\end{aligned}
$$

and

$$
\lim _{t \longrightarrow \infty} \varphi_{2}(t)=0 .
$$

On the other hand, we will discuss the long time behavior of the microorganism $x$ in two cases, we define

$$
V=\ln x
$$

Case 1. If $S(t)<1$, we get

$$
\begin{aligned}
\mathrm{d} V & =\left(\frac{m S}{k x+S}-D-\frac{1}{2} \sigma_{2}^{2}\right) \mathrm{d} t+\sigma_{2} \mathrm{~d} B_{2}(t) \\
& \geq\left(\frac{m S}{k H_{2}+1}-D-\frac{1}{2} \sigma_{2}^{2}\right) \mathrm{d} t+\sigma_{2} \mathrm{~d} B_{2}(t),
\end{aligned}
$$

so,

$$
\frac{V(t)}{t}-\frac{V(0)}{t} \geq \frac{m}{k H_{2}+1}\langle S(t)\rangle-D-\frac{1}{2} \sigma_{2}^{2}+\frac{\sigma_{2}}{t} B_{2}(t) .
$$




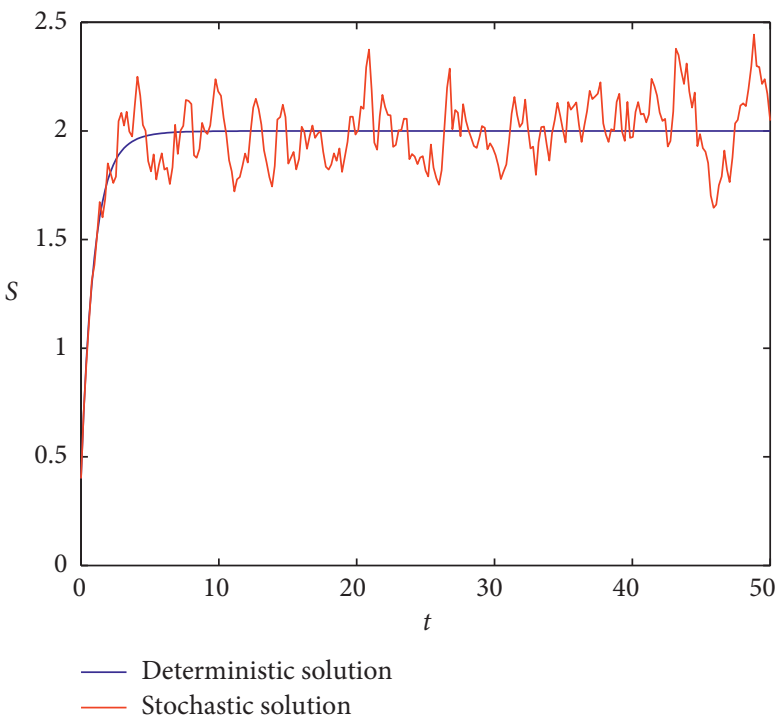

(a)

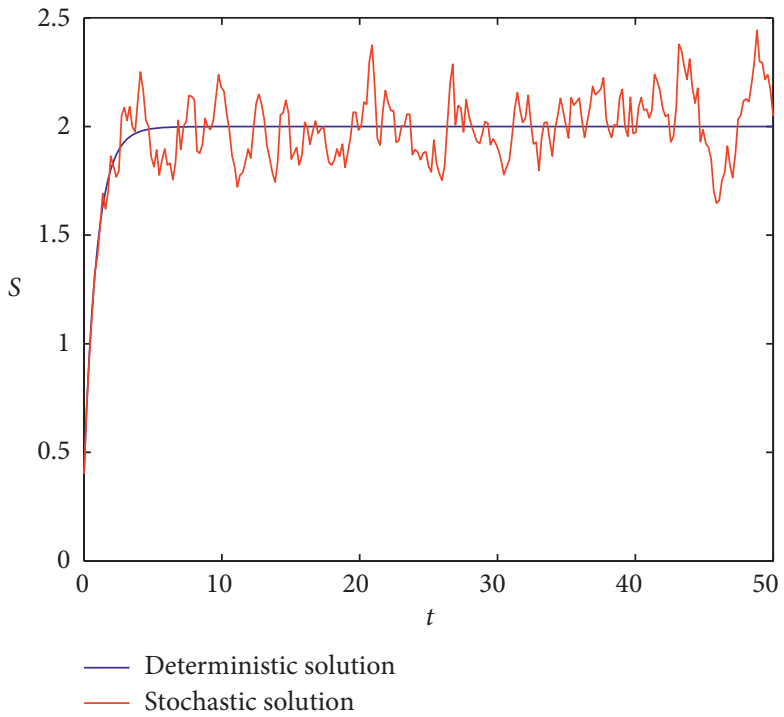

(c)

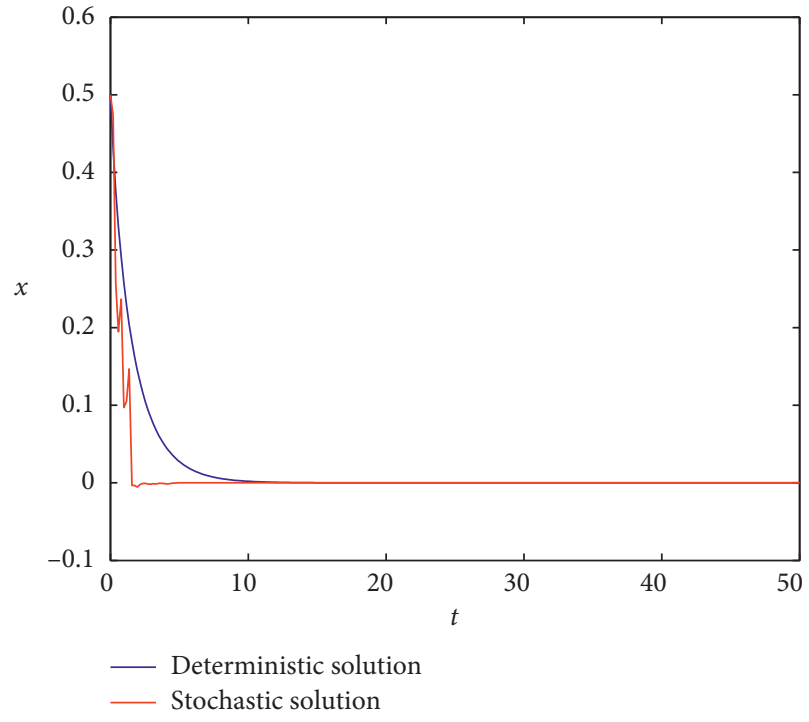

(b)

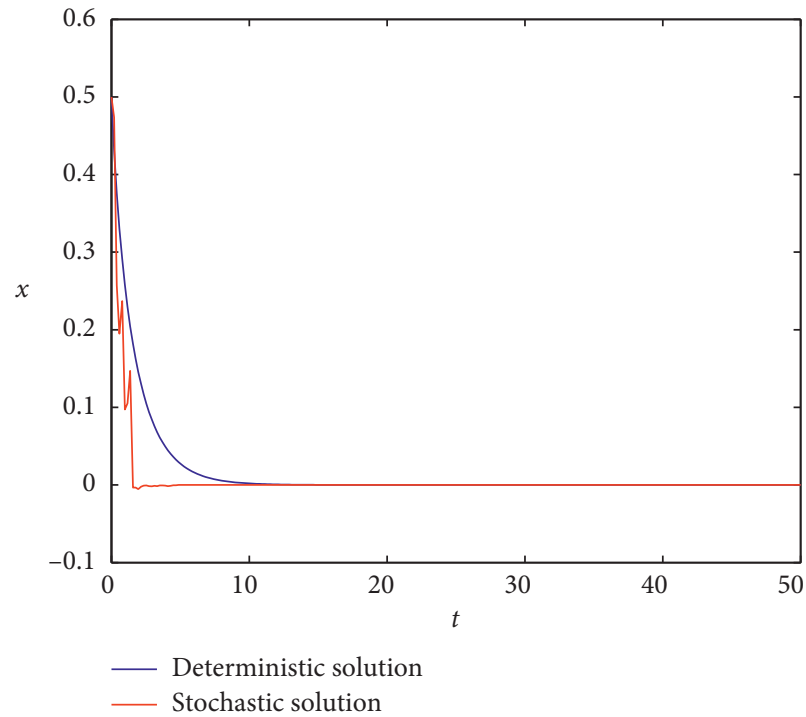

(d)

Figure 1: The solutions of deterministic model (3) and stochastic model (4). (a) Sample paths of $S(t)$ with $A=2, B=0$. (b) Sample paths of $x(t)$ with $A=2, B=0$. (c) Sample paths of $S(t)$ with $A=2, B=3$. (d) Sample paths of $x(t)$ with $A=2, B=3$.

Substituting (39) into (45), we obtain

$$
\begin{aligned}
\frac{V(t)}{t}-\frac{V(0)}{t} \geq & \frac{m}{k H_{2}+1}\left(S^{0}-\frac{m+A \quad D}{A \quad D}\langle x(t)\rangle-\varphi_{2}(t)\right) \\
& -D-\frac{1}{2} \sigma_{2}^{2}+\frac{\sigma_{2}}{t} B_{2}(t) .
\end{aligned}
$$

When $S^{0}<1$, we have

$$
\begin{aligned}
\ln x(t) \geq & \left(\frac{m S^{0}}{k H_{2}+1}-D-\frac{1}{2} \sigma_{2}^{2}\right) t-\frac{m(m+A \quad D)}{A D\left(k H_{2}+1\right)}\langle x(t)\rangle t \\
& \quad-\frac{m t \varphi_{2}(t)}{k H_{2}+1}+\sigma_{2} B_{2}(t)+\ln x_{0} \\
\triangleq & \lambda_{1} t-\lambda_{0} \int_{0}^{t} x(s) \mathrm{d} s+F_{1}(t),
\end{aligned}
$$




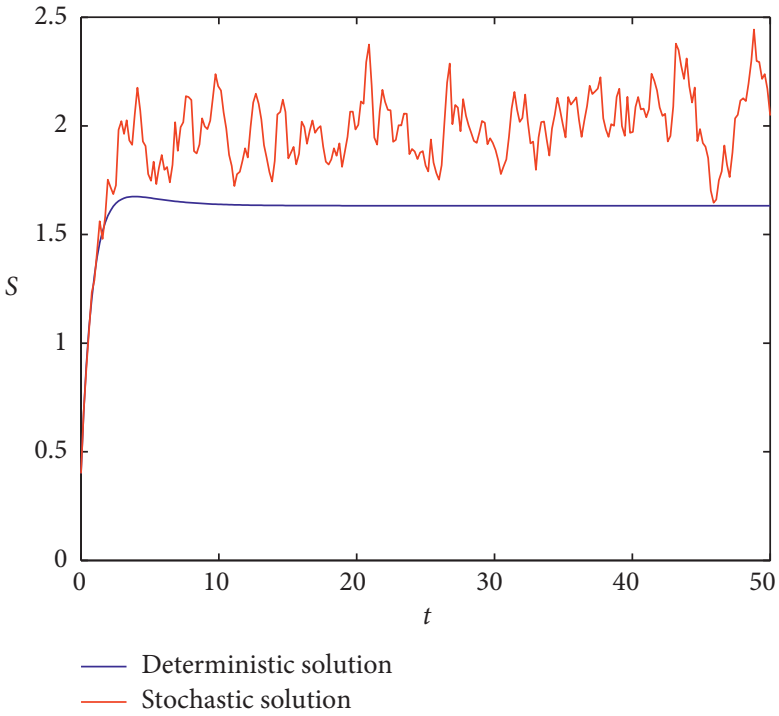

(a)

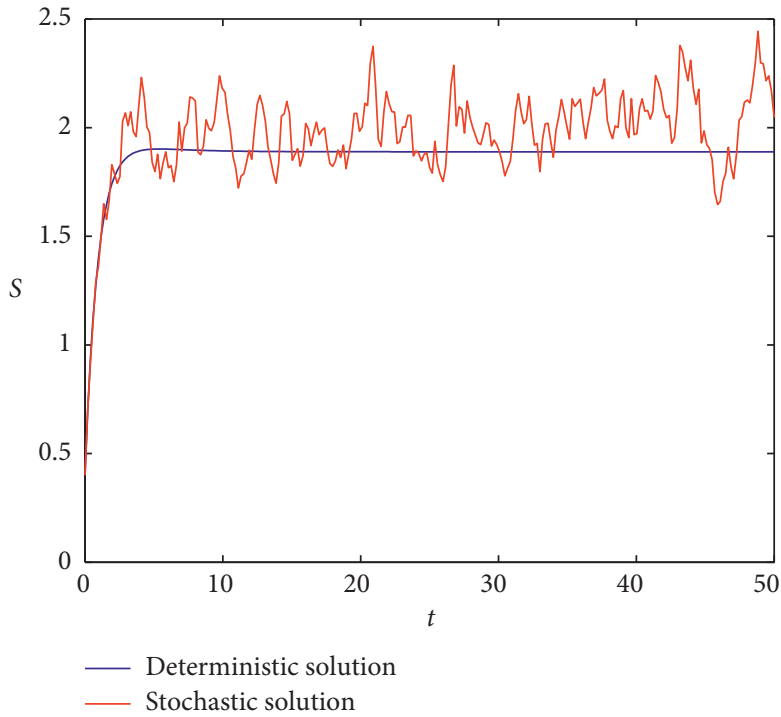

(c)

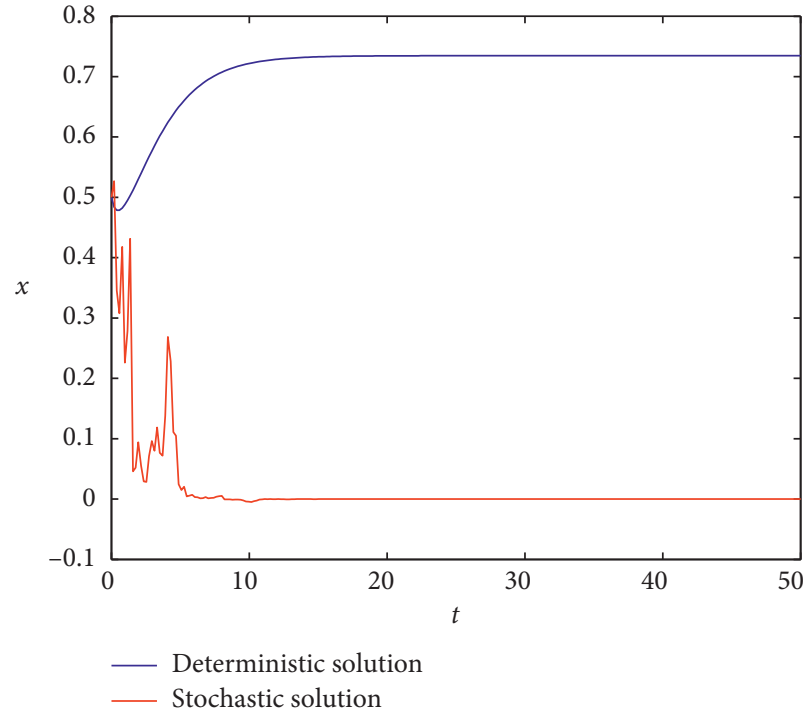

(b)

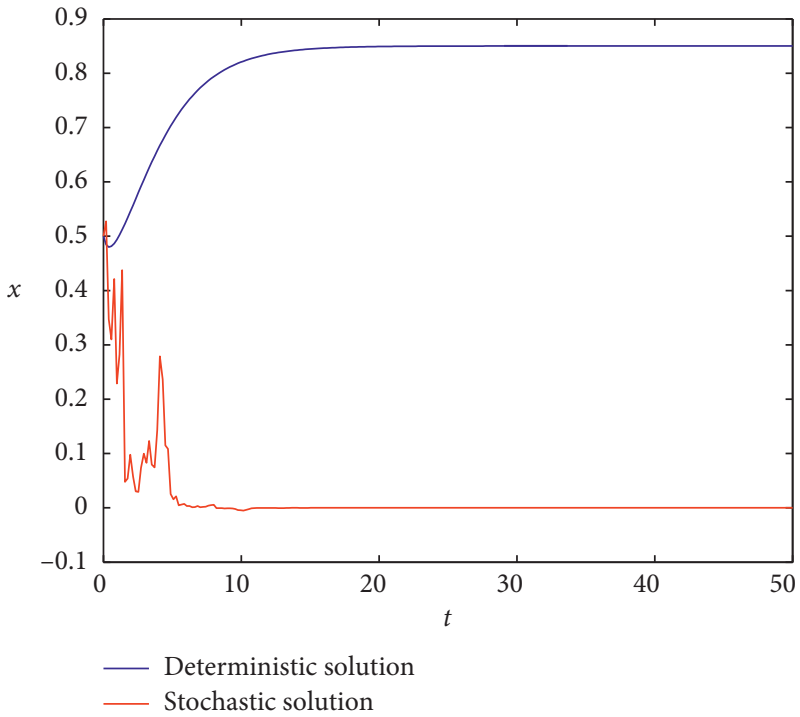

(d)

Figure 2: The solutions of deterministic model (3) and stochastic model (4). (a) Sample paths of $S(t)$ with $A=2, B=0$. (b) Sample paths of $x(t)$ with $A=2, B=0$. (c) Sample paths of $S(t)$ with $A=2, B=3$. (d) Sample paths of $x(t)$ with $A=2, B=3$.

$$
\begin{aligned}
\lambda_{1} & =\frac{m S^{0}}{k H_{2}+1}-D-\frac{1}{2} \sigma_{2}^{2}, \\
\lambda_{0} & =\frac{m(m+A D)}{A D\left(k H_{2}+1\right)}, \\
F_{1}(t) & =-\frac{m t \varphi_{2}(t)}{k H_{2}+1}+\sigma_{2} B_{2}(t)+\ln x_{0},
\end{aligned}
$$

take note of the condition of Theorem 3, we have $\lambda_{1}>0$. Thus, from (42), Lemmas 3 and 4, we can obtain that

$$
\langle x(t)\rangle_{*} \geq \frac{A \quad D\left(k H_{2}+1\right)}{m(m+A D)}\left(\frac{m S^{0}}{k H_{2}+1}-D-\frac{1}{2} \sigma_{2}^{2}\right)>0 .
$$

When $S^{0} \geq 1$, we have

$$
\begin{aligned}
\ln x(t) \geq & \left(\frac{m}{k H_{2}+1}-D-\frac{1}{2} \sigma_{2}^{2}\right) t-\frac{m(m+A D)}{A D\left(k H_{2}+1\right)}\langle x(t)\rangle t \\
& \quad-\frac{m t \varphi_{2}(t)}{k H_{2}+1}+\sigma_{2} B_{2}(t)+\ln x_{0} \\
\triangleq & \lambda_{2} t-\lambda_{0} \int_{0}^{t} x(s) \mathrm{d} s+F_{1}(t),
\end{aligned}
$$

where $\lambda_{2}=\left(m / k H_{2}+1\right)-D-(1 / 2) \sigma_{2}^{2}$, similar to the above, we can obtain that 


$$
\langle x(t)\rangle_{*} \geq \frac{A D\left(k H_{2}+1\right)}{m(m+A D)}\left(\frac{m}{k H_{2}+1}-D-\frac{1}{2} \sigma_{2}^{2}\right)>0 .
$$

Case 2. If $S(t) \geq 1$, we get

$$
\begin{aligned}
\mathrm{d} V & =\left(\frac{m S}{k x+S}-D-\frac{1}{2} \sigma_{2}^{2}\right) \mathrm{d} t+\sigma_{2} \mathrm{~d} B_{2}(t) \\
& \geq\left(\frac{m}{k H_{2}+1}-D-\frac{1}{2} \sigma_{2}^{2}\right) \mathrm{d} t+\sigma_{2} \mathrm{~d} B_{2}(t),
\end{aligned}
$$

so,

$$
\frac{V(t)}{t}-\frac{V(0)}{t} \geq \frac{m}{k H_{2}+1}-D-\frac{1}{2} \sigma_{2}^{2}+\frac{\sigma_{2}}{t} B_{2}(t) .
$$

Obviously,

$$
\begin{aligned}
\frac{V(t)}{t}-\frac{V(0)}{t} \geq & \frac{m}{k H_{2}+1}-D-\frac{1}{2} \sigma_{2}^{2}-\frac{m(m+A \quad D)}{A D\left(k H_{2}+1\right)}\langle x(t)\rangle t \\
& +\frac{\sigma_{2}}{t} B_{2}(t) .
\end{aligned}
$$

When $S^{0}<1$, we have

$$
\begin{aligned}
\ln x(t) \geq & \left(\frac{m S^{0}}{k H_{2}+1}-D-\frac{1}{2} \sigma_{2}^{2}\right) t-\frac{m(m+A \quad D)}{A\left(k H_{2}+1\right)}\langle x(t)\rangle t \\
& +\sigma_{2} B_{2}(t)+\ln \quad x_{0} \\
\triangleq & \lambda_{1} t-\lambda_{0} \int_{0}^{t} x(s) \mathrm{d} s+F_{2}(t)
\end{aligned}
$$

where $F_{2}(t)=\sigma_{2} B_{2}(t)+\ln x_{0}$, similarly, we have

$$
\langle x(t)\rangle_{*} \geq \frac{A \quad D\left(k H_{2}+1\right)}{m(m+A D)}\left(\frac{m S^{0}}{k H_{2}+1}-D-\frac{1}{2} \sigma_{2}^{2}\right)>0 .
$$

When $S^{0} \geq 1$, we have

$$
\begin{aligned}
\ln x(t) \geq & \left(\frac{m}{k H_{2}+1}-D-\frac{1}{2} \sigma_{2}^{2}\right) t-\frac{m(m+A \quad D)}{A \quad D\left(k H_{2}+1\right)}\langle x(t)\rangle t \\
& +\sigma_{2} B_{2}(t)+\ln x_{0} \\
\triangleq & \lambda_{2} t-\lambda_{0} \int_{0}^{t} x(s) \mathrm{d} s+F_{2}(t)
\end{aligned}
$$

Similar to the above, we have

$$
\langle x(t)\rangle_{*} \geq \frac{A \quad D\left(k H_{2}+1\right)}{m(m+A D)}\left(\frac{m}{k H_{2}+1}-D-\frac{1}{2} \sigma_{2}^{2}\right)>0 .
$$

In conclusion, no matter what $S(t)$ chooses, we have

$$
\langle x(t)\rangle_{*}>0 \text {. }
$$

This completes the proof of Theorem 3 .

Remark 3. It follows from Theorem 3 that the microorganism will be strongly persistent in the mean if the condition $(1 / 2) \sigma_{2}^{2}<\zeta \triangleq \min \left\{\left(m / k H_{2}+1\right)-D,\left(m S^{0} / k H_{2}+1\right)-\right.$ $D\}$ is satisfied. That is, when the intensity of the white noise is small enough, the microorganism will survive (see Figures 3 and 4).

Stationary distribution is one of the most significant dynamical characteristics of the stochastic model; that is, the stochastic model has a stationary distribution which represents the persistence of microorganism in the future. Therefore, in the rest of this section, we will make a positive decision by the existence of the stationary distribution. Before proving the main result, several known results are given for the stochastic differential equations.

Assume $X(t)$ is a regular time-homogeneous Markov Process in $n$-dimensional Euclidean space $E^{n}$. The stochastic differential equation takes the following form:

$$
\mathrm{d} X(t)=h(X) \mathrm{d} t+\sum_{r=1}^{n} g_{r}(X) \mathrm{d} B_{r}(t) .
$$

The diffusion matrix of the process $X(t)$ is defined as follows:

$$
\begin{aligned}
\mathscr{A}(x) & =\left(\alpha_{i j}(x)\right) \\
\alpha_{i j}(x) & =\sum_{r=1}^{n} g_{r}^{i}(x) g_{r}^{j}(x) .
\end{aligned}
$$

Define the differential operator $L$ associated with (60) by

$$
L=\frac{\partial}{\partial t}+\sum_{i=1}^{n} h_{i}(x) \frac{\partial}{\partial x_{i}}+\frac{1}{2} \sum_{i, j=1}^{n} \alpha_{i j}(x) \frac{\partial^{2}}{\partial x_{i} \partial x_{j}} .
$$

Lemma 5 (see [30]). Assume that there exists a bounded open domain $U \subset E^{n}$ with regular boundary $\Gamma$, having the following properties:

(1) In the domain $U$ and some neighborhood thereof, the smallest eigenvalue of the diffusion matrix $\mathscr{A}$ is bounded away from zero.

(2) If $x \in E^{n} \backslash U$, the mean time $\tau$ at which a path issuing from $x$ reaches the set $U$ is finite, and $\sup _{x \in K} E^{x} \tau<\infty$ for every compact subset $K \subset E^{n}$.

Then the Markov process $X(t)$ has a stationary distribution $\mu(\cdot)$, and it is unique.

Remark 4. Since we investigate the stochastic model (4) in the space $\mathbb{R}_{+}^{2}$, to validate condition (1) of Lemma 5 , we need to prove that for any bounded open domain $U \subset \mathbb{R}_{+}^{2}$, there is a positive number $M_{0}$ such that $\sum_{i, j=1}^{2} \alpha_{i j}(x) \xi_{i} \xi_{j} \geq M_{0}|\xi|^{2}$, $x \in \bar{U}, \xi \in \mathbb{R}_{+}^{2}$ (see $[31,32]$ ). To validate condition (2) of Lemma 5, we need to show that there exists a neighborhood $U$ and a non-negative $C^{2}$-function $V$ such that for any $(S, x) \in \mathbb{R}_{+}^{2} \backslash U, L V$ is negative. 


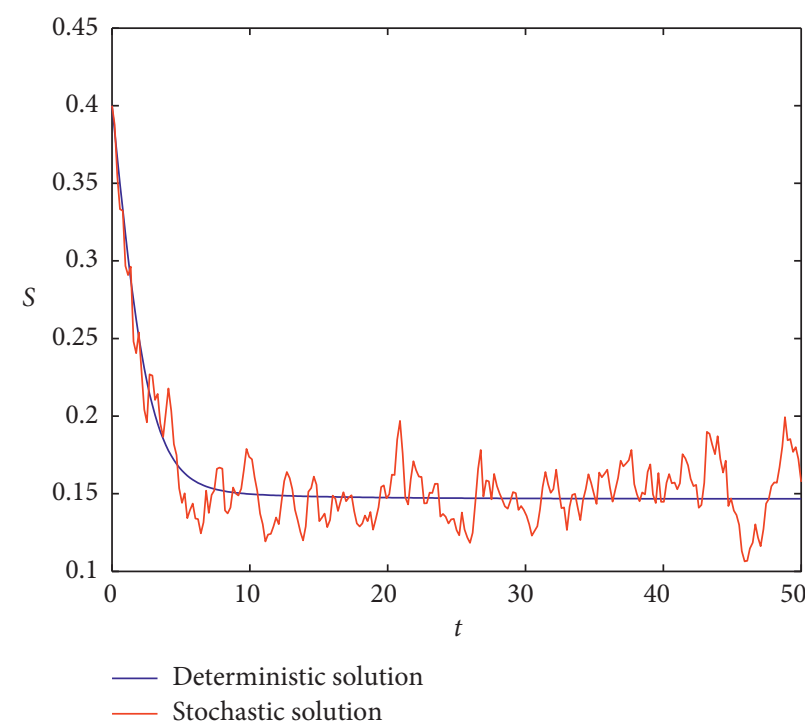

(a)

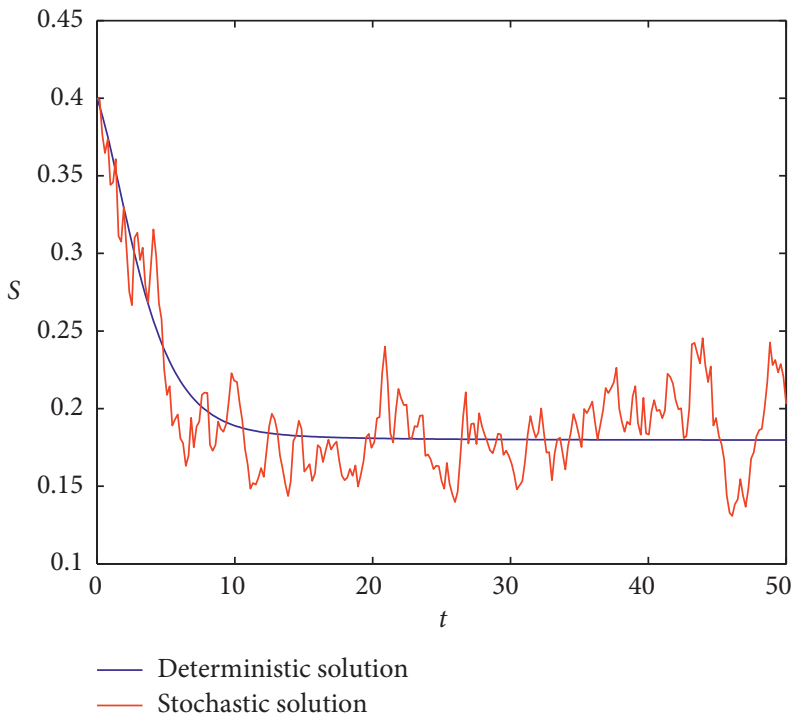

(c)

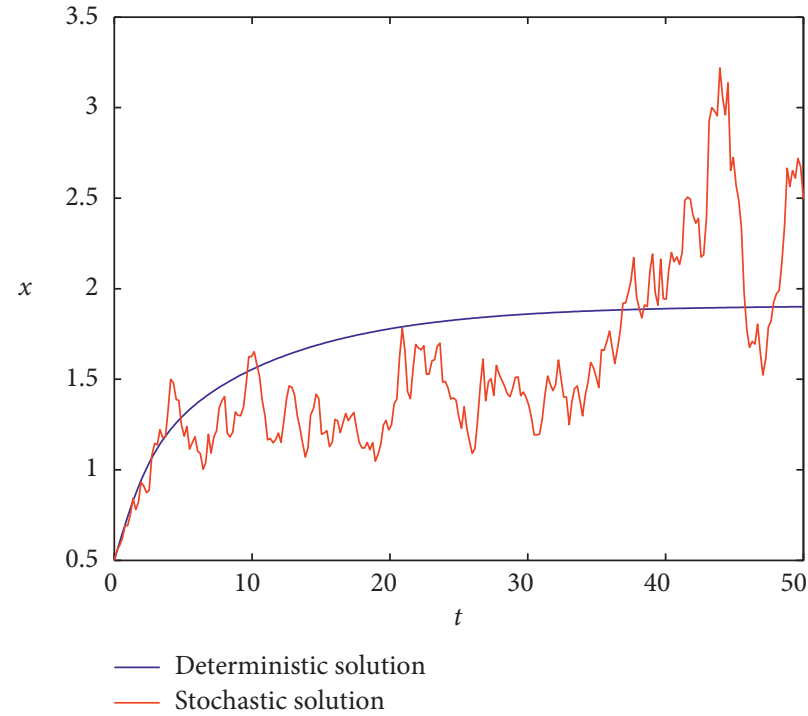

(b)

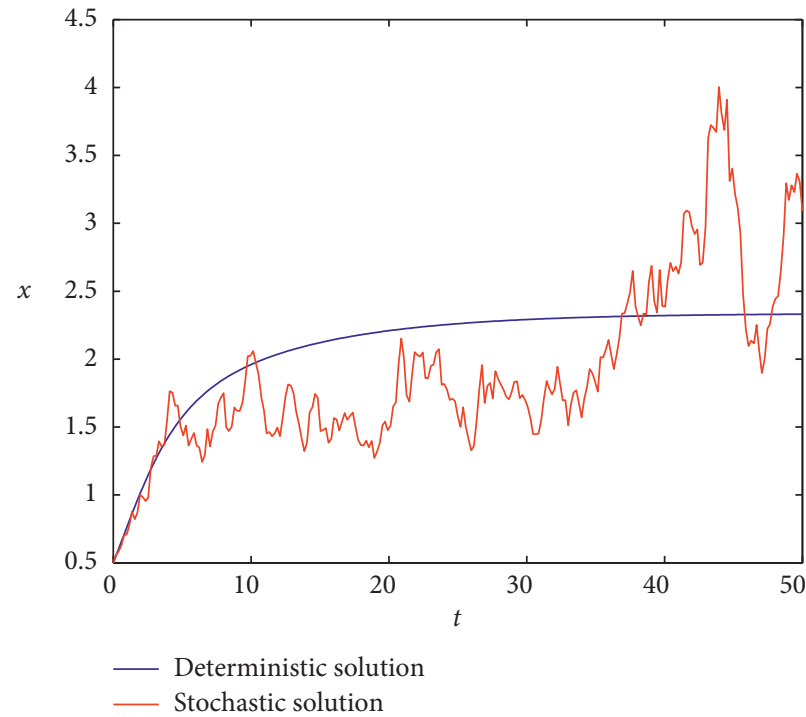

(d)

Figure 3: The solutions of deterministic model (3) and stochastic model (4). (a) Sample paths of $S(t)$ with $A=2, B=0$. (b) Sample paths of $x(t)$ with $A=2, B=0$. (c) Sample paths of $S(t)$ with $A=2, B=3$. (d) Sample paths of $x(t)$ with $A=2, B=3$.

The following theorem shows that the model (4) has a unique stationary distribution for any given initial value (5).

Theorem 4. Let $\eta=c_{2} S^{0} D-\left(c_{1} m S^{0} / k A\right)-\left(c_{1} \sigma_{1}^{2} S^{0} / 2\right)-D-$ $\left(\sigma_{2}^{2} / 2\right)$ where $c_{2}=\left(m k H_{2} / D\left(k H_{2}+S^{0}\right)^{2}\right)$. If there exists a constant $c_{1}>\left(m k H_{2} S^{0} / D\left(k H_{2}+S^{0}\right)^{3}\right)$ such that $\eta$ is positive, then for any given initial value (5), the solution of the model (4) admits a unique stationary distribution $\mu(\cdot)$.

Proof. According to the model (4), we get

$$
\begin{aligned}
& d\left(\begin{array}{l}
S \\
x
\end{array}\right)=\left(\begin{array}{c}
\left(S^{0}-S\right) D-\frac{m S x}{(k x+S)(A+B S)} \\
\frac{m S x}{k x+S}-x \quad D
\end{array}\right) \mathrm{d} t \\
& +\left(\begin{array}{cc}
\sigma_{1} S & 0 \\
0 & \sigma_{2} x
\end{array}\right)\left(\begin{array}{c}
\mathrm{d} B_{1}(t) \\
\mathrm{d} B_{2}(t)
\end{array}\right) .
\end{aligned}
$$

So, the diffusion matrix is 


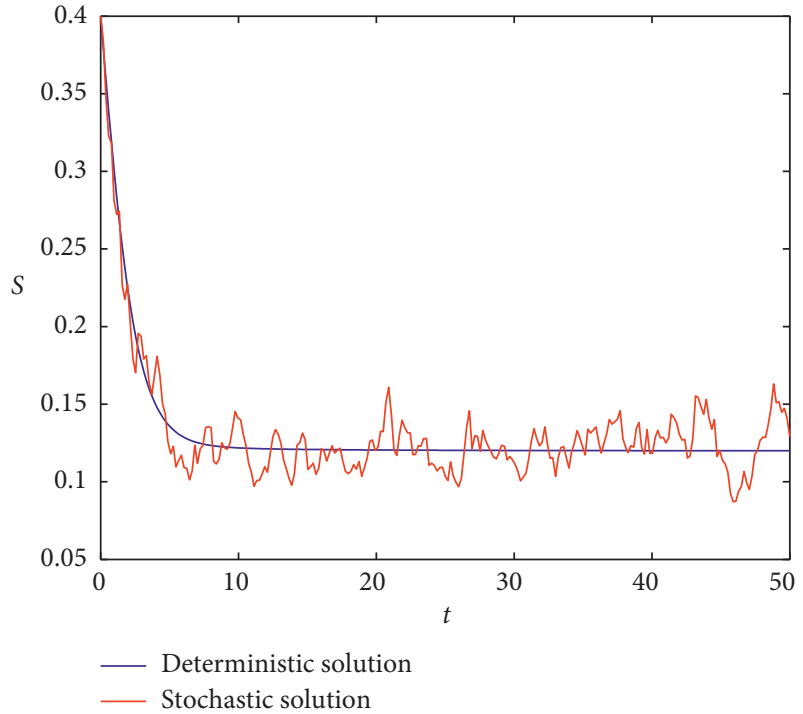

(a)

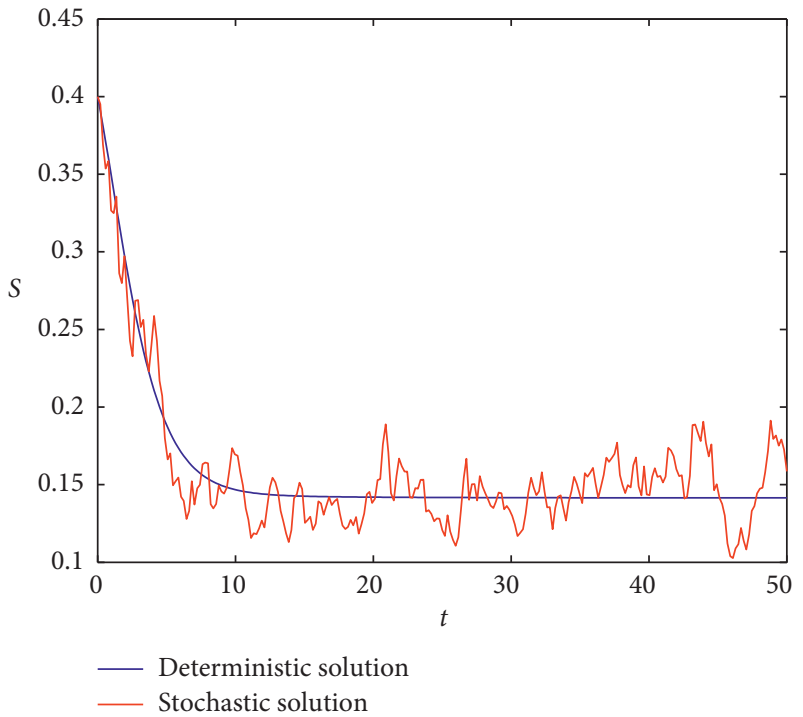

(c)

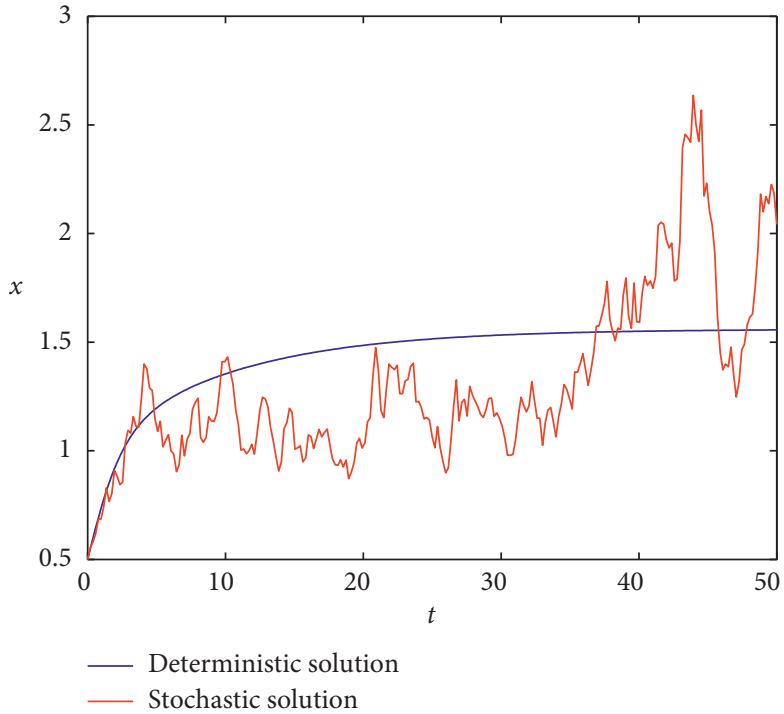

(b)

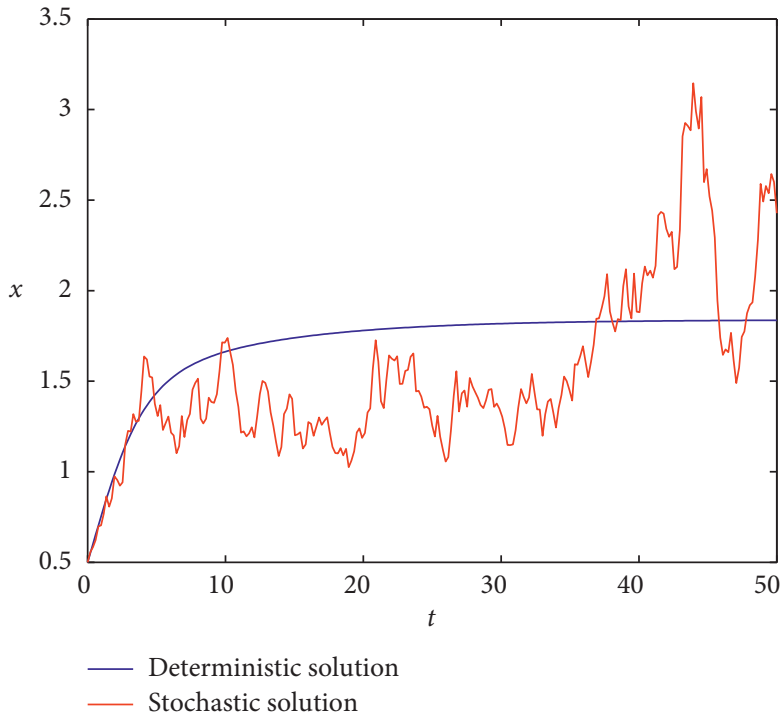

(d)

Figure 4: The solutions of deterministic model (3) and stochastic model (4). (a) Sample paths of $S(t)$ with $A=2, B=0$. (b) Sample paths of $x(t)$ with $A=2, B=0$. (c) Sample paths of $S(t)$ with $A=2, B=3$. (d) Sample paths of $x(t)$ with $A=2, B=3$.

$$
\mathscr{A}(S, x)=\left(\begin{array}{cc}
\sigma_{1}^{2} S^{2} & 0 \\
0 & \sigma_{2}^{2} x^{2}
\end{array}\right)
$$

Let $U$ be any bounded open domain in $\mathbb{R}_{+}^{2}$, then there exists a positive constant

$$
M_{0}=\min \left\{\sigma_{1}^{2} S^{2}, \sigma_{2}^{2} x^{2},(S, x) \in \bar{U}\right\},
$$

such that

$$
\sum_{i, j=1}^{2} \alpha_{i j}(S, x) \xi_{i} \xi_{j}=\sigma_{1}^{2} S^{2} \xi_{1}^{2}+\sigma_{2}^{2} x^{2} \xi_{2}^{2} \geq M_{0}|\xi|^{2},
$$

for all $(S, x) \in \bar{U}, \xi \in \mathbb{R}_{+}^{2}$, which implies that condition (1) of Lemma 5 is satisfied.
Next, we need to construct a non-negative $C^{2}$-function $V(S, x)$ and a open set $U \in \mathbb{R}_{+}^{2}$, such that

$$
\sup _{(S, x) \in \mathbb{R}_{+}^{2} \backslash U} L V(S, x)<0 .
$$

Then, we select $\theta \in(0,1)$ to be a sufficiently small constant such that

$$
\text { (A.1) } D-\frac{\theta}{2}\left(\sigma_{1}^{2} \vee \sigma_{2}^{2}\right)>0,
$$

choosing a large enough positive constant $N$ such that

$$
\text { (A.2) } \psi_{1}^{u}-N \eta \leq-2 \text {, }
$$

where $\psi_{1}^{u}=\sup _{(S, x) \in \mathbb{R}_{+}^{2} \backslash U} \psi_{1}(S, x)$, and $\psi_{1}(S, x)$ is given in (A.3). 
Define a nonnegative $C^{2}$-Lyapunov function

$$
V(S, x)=H(S, x)-H\left(S_{*}, x_{*}\right),
$$

where $H(S, x)=N V_{1}+V_{2}+V_{3}, V_{1}=c_{1}\left(S-S^{0}-S^{0} \ln \left(S / S^{0}\right)\right)$ $\ln x-c_{2}(S+x), V_{2}=(1 /(\theta+1))(S+x)^{\theta+1}, V_{3}=-\ln S$, and

$$
\begin{aligned}
\mathrm{d}\left(S-S^{0}-S^{0} \ln \frac{S}{S^{0}}\right) & =\left(-\frac{\left(S-S^{0}\right)^{2} D}{S}-\frac{m x\left(S-S^{0}\right)}{(k x+S)(A+B S)}+\frac{1}{2} \sigma_{1}^{2} S^{0}\right) \mathrm{d} t+\sigma_{1}\left(S-S^{0}\right) \mathrm{d} B_{1}(t), \\
\mathrm{d}(\ln x) & =\left(\frac{m S}{k x+S}-D-\frac{1}{2} \sigma_{2}^{2}\right) \mathrm{d} t+\sigma_{2} \mathrm{~d} B_{2}(t), \\
\mathrm{d}(S+x) & =\left(\begin{array}{ll}
\left(S^{0}-S\right) D-\frac{m S x}{(k x+S)(A+B S)}+\frac{m S x}{k x+S}-x \quad D
\end{array}\right) \mathrm{d} t+\sigma_{1} S \mathrm{~d} B_{1}(t)+\sigma_{2} x \mathrm{~d} B_{2}(t) .
\end{aligned}
$$

So,

$$
\begin{aligned}
\mathrm{d} V_{1}= & c_{1}\left(-\frac{\left(S-S^{0}\right)^{2} D}{S}-\frac{m x\left(S-S^{0}\right)}{(k x+S)(A+B S)}+\frac{1}{2} \sigma_{1}^{2} S^{0}\right) \mathrm{d} t-\left(\frac{m S}{k x+S}-D-\frac{1}{2} \sigma_{2}^{2}\right) \mathrm{d} t \\
& -c_{2}\left(\left(S^{0}-S\right) D-\frac{m S x}{(k x+S)(A+B S)}+\frac{m S x}{k x+S}-x \quad D\right) \mathrm{d} t \\
& +c_{1} \sigma_{1}\left(S-S^{0}\right) \mathrm{d} B_{1}(t)-\sigma_{2} \mathrm{~d} B_{2}(t)-c_{2}\left(\sigma_{1} S \mathrm{~d} B_{1}(t)+\sigma_{2} x \mathrm{~d} B_{2}(t)\right),
\end{aligned}
$$

thus,

$$
\begin{aligned}
L V_{1}= & -\frac{c_{1} D\left(S-S^{0}\right)^{2}}{S}-\frac{c_{1} m x\left(S-S^{0}\right)}{(k x+S)(A+B S)}+\frac{c_{1}}{2} \sigma_{1}^{2} S^{0}-\frac{m S}{k x+S}+D+\frac{1}{2} \sigma_{2}^{2} \\
& -c_{2} D\left(S^{0}-S\right)+\frac{c_{2} m S x}{(k x+S)(A+B S)}-\frac{c_{2} m S x}{k x+S}+c_{2} D x \\
\leq & -\frac{c_{1} D\left(S-S^{0}\right)^{2}}{S}+\frac{c_{1} m S^{0}}{k A}+\frac{c_{1}}{2} \sigma_{1}^{2} S^{0}-\frac{m S}{k H_{2}+S}+D+\frac{1}{2} \sigma_{2}^{2} \\
= & \left(-\frac{c_{1} D\left(S-S^{0}\right)^{2}}{S}-\frac{m S}{k H_{2}+S}+c_{2} D S\right)+\left(\frac{c_{2} m}{A}+c_{2} D\right) x \\
& -\left(c_{2} D S^{0}-\frac{c_{1} m S^{0}}{k A}-\frac{c_{1}}{2} \sigma_{1}^{2} S^{0}-D-\frac{1}{2} \sigma_{2}^{2}\right) \\
\triangleq & l(S)+\left(\frac{c_{2} m}{A}+c_{2} D\right) x-\eta,
\end{aligned}
$$


where

$$
\begin{gathered}
l(S)=-\frac{c_{1} D\left(S-S^{0}\right)^{2}}{S}-\frac{m S}{k H_{2}+S}+c_{2} D S, \\
\eta=c_{2} D S^{0}-\frac{c_{1} m S^{0}}{k A}-\frac{c_{1}}{2} \sigma_{1}^{2} S^{0}-D-\frac{1}{2} \sigma_{2}^{2} .
\end{gathered}
$$
have

Therefore, according to the previous function $l(S)$, we

$$
\begin{aligned}
& l^{\prime}(S)=-\frac{c_{1} D\left(S^{2}-S^{02}\right)}{S^{2}}-\frac{m k H_{2}}{\left(k H_{2}+S\right)^{2}}+c_{2} D, \\
& l^{\prime \prime}(S)=-\frac{2 c_{1} D S^{02}}{S^{3}}+\frac{2 m k H_{2}}{\left(k H_{2}+S\right)^{3}} .
\end{aligned}
$$

Obviously,

$$
\begin{aligned}
& \left.l \prime(S)\right|_{S=S^{0}}=0, \quad \text { for } c_{2}=\frac{m k H_{2}}{D\left(k H_{2}+S^{0}\right)^{2}}, \\
& \left.l^{\prime \prime}(S)\right|_{S=S^{0}}=\frac{-2 c_{1} D\left(k H_{2}+S^{0}\right)^{3}+2 m k H_{2} S^{0}}{S^{0}\left(k H_{2}+S^{0}\right)^{3}}<0, \\
& \text { for } c_{1}>\frac{m k H_{2} S^{0}}{D\left(k H_{2}+S^{0}\right)^{3}} .
\end{aligned}
$$

So,

$$
l(S) \leq l\left(S^{0}\right)=c_{2} D S^{0}-\frac{m S^{0}}{k H_{2}+S^{0}}=-\frac{m S^{02}}{\left(k H_{2}+S^{0}\right)^{2}}<0,
$$

thus,

$$
L V_{1} \leq-\eta+\left(\frac{c_{2} m}{A}+c_{2} D\right) x
$$

Now, we estimate $L V_{2}$ and $L V_{3}$,

$$
\begin{aligned}
\mathrm{d} V_{2}= & (S+x)^{\theta}\left(\left(S^{0}-S\right) D-\frac{m S x}{(k x+S)(A+B S)}\right. \\
& \left.+\frac{m S x}{k x+S}-x D\right) \mathrm{d} t \\
& +\frac{\theta}{2}(S+x)^{\theta-1}\left(\sigma_{1}^{2} S^{2}+\sigma_{2}^{2} x^{2}\right) \mathrm{d} t+(S+x)^{\theta}\left(\sigma_{1} S \mathrm{~d} B_{1}(t)\right. \\
& \left.+\sigma_{2} x \mathrm{~d} B_{2}(t)\right) \\
= & L V_{2} \mathrm{~d} t+(S+x)^{\theta}\left(\sigma_{1} S \mathrm{~d} B_{1}(t)+\sigma_{2} x \mathrm{~d} B_{2}(t)\right) .
\end{aligned}
$$

So,

$$
\begin{aligned}
L V_{2}= & (S+x)^{\theta}\left(\left(S^{0}-S\right) D-\frac{m S x}{(k x+S)(A+B S)}\right. \\
& \left.+\frac{m S x}{k x+S}-x D\right)+\frac{\theta}{2}(S+x)^{\theta-1}\left(\sigma_{1}^{2} S^{2}+\sigma_{2}^{2} x^{2}\right) \\
\leq & (S+x)^{\theta} S^{0} D-(S+x)^{\theta+1} D+m H_{2}(S+x)^{\theta} \\
& +\frac{\theta}{2}(S+x)^{\theta-1}\left(\sigma_{1}^{2} \vee \sigma_{2}^{2}\right)(S+x)^{2} \\
= & \left(S^{0} D+m H_{2}\right)(S+x)^{\theta}-\left(D-\frac{\theta}{2}\left(\sigma_{1}^{2} \vee \sigma_{2}^{2}\right)\right)(S+x)^{\theta+1},
\end{aligned}
$$

where the positive constant $H_{2}$ is mentioned in Lemma 2.

$$
\begin{aligned}
\mathrm{d} V_{3}= & -\frac{1}{S}\left(S^{0} D-S D-\frac{m S x}{(k x+S)(A+B S)}\right) \mathrm{d} t+\frac{1}{2} \sigma_{1}^{2} \mathrm{~d} t \\
& -\sigma_{1} \mathrm{~d} B_{1}(t) \\
= & L V_{3} \mathrm{~d} t-\sigma_{1} \mathrm{~d} B_{1}(t) .
\end{aligned}
$$

So,

$$
\begin{aligned}
L V_{3} & =-\frac{S^{0} D}{S}+D+\frac{m x}{(k x+S)(A+B S)}+\frac{1}{2} \sigma_{1}^{2} \\
& \leq-\frac{S^{0} D}{S}+D+\frac{m}{k A}+\frac{1}{2} \sigma_{1}^{2},
\end{aligned}
$$

thus, from $V(S, x)=N V_{1}+V_{2}+V_{3}-H\left(S_{*}, x_{*}\right)$, we have

$$
\begin{aligned}
L V \leq & N\left(-\eta+\left(\frac{c_{2} m}{A}+c_{2} D\right) x\right)+\left(S^{0} D+m H_{2}\right)(S+x)^{\theta} \\
& -\left(D-\frac{\theta}{2}\left(\sigma_{1}^{2} \vee \sigma_{2}^{2}\right)\right)(S+x)^{\theta+1} \\
& -\frac{S^{0} D}{S}+D+\frac{m}{k A}+\frac{1}{2} \sigma_{1}^{2} \\
\triangleq & \psi_{1}(S, x)+\psi_{2}(x),
\end{aligned}
$$

where 
(A.3)

$$
\left\{\begin{array}{l}
\psi_{1}(S, x)=\left(S^{0} D+m H_{2}\right)(S+x)^{\theta}-\left(D-\frac{\theta}{2}\left(\sigma_{1}^{2} \vee \sigma_{2}^{2}\right)\right)(S+x)^{\theta+1}-\frac{S^{0} D}{S}+D+\frac{m}{k A}+\frac{1}{2} \sigma_{1}^{2}, \\
\psi_{2}(x)=N\left(-\eta+\left(\frac{c_{2} m}{A}+c_{2} D\right) x\right) .
\end{array}\right.
$$

$$
M(t)=\int_{0}^{t} \mathscr{M}(t) \mathrm{d} t
$$

Through observation, we find that

$$
\begin{aligned}
& \psi_{1}(S, x)+\psi_{2}(x)=-\infty, \quad \text { as } S \longrightarrow+\infty, x \in \mathbb{R}^{+}, \\
& \psi_{1}(S, x)+\psi_{2}(x)=-\infty, \quad \text { as } x \longrightarrow+\infty, S \in \mathbb{R}^{+}, \\
& \psi_{1}(S, x)+\psi_{2}(x)=-\infty, \quad \text { as } S \longrightarrow 0^{+}, x \in \mathbb{R}^{+}, \\
& \psi_{1}(S, x)+\psi_{2}(x) \leq-2, \quad \text { as } x \longrightarrow 0^{+}, S \in \mathbb{R}^{+} .
\end{aligned}
$$

Let $U=(\varepsilon,(1 / \varepsilon)) \times(\varepsilon,(1 / \varepsilon))$, where $\varepsilon$ is a small enough constant. It follows that

$$
L V<0, \quad(S, x) \in \mathbb{R}_{+}^{2} \backslash U .
$$

This implies that the model (4) admits a unique stationary distribution $\mu(\cdot)$.

Thus, the proof is completed.

Remark 5. By Theorem 4, we known that the model (4) has a unique stationary distribution if the condition holds, which means that the microorganism is persistent in the future.

\section{Stochastic Maximum Principle}

In this section, the existence of the optimal stochastic control for the model (4) is proved. Our aim is to find an optimal stochastic control under given the initial conditions such that the production of the microorganism is maximized at a given time $T$. We select the dilution rate $D$ as stochastic control variable $D(t)$. Several preliminaries are given.

For convenience, the model (4) can be rewritten as

$$
\left\{\begin{array}{l}
\mathrm{d} S(t)=f_{1}(S(t), x(t), D(t)) \mathrm{d} t+\sigma_{1} S(t) \mathrm{d} B_{1}(t), \\
\mathrm{d} x(t)=f_{2}(S(t), x(t), D(t)) \mathrm{d} t+\sigma_{2} x(t) \mathrm{d} B_{2}(t),
\end{array}\right.
$$

where

$$
\begin{aligned}
f_{1}(S(t), x(t), D(t))= & \left(S^{0}-S(t)\right) D(t)-\frac{m S(t)}{k x(t)+S(t)} \\
& \cdot \frac{x(t)}{A+B S(t)}, \\
f_{2}(S(t), x(t), D(t))= & x(t)\left(\frac{m S(t)}{k x(t)+S(t)}-D(t)\right) .
\end{aligned}
$$

The stochastic control variable $D(t)$ is a non-negative continuous bounded function. Let the constant $V$ denote the volume of the culture vessel, and let $F(t)$ be the volumetric flow rate, then the dilution rate $D(t)=(F(t) / V)$. For convenience, we define $V=1$, so $D(t)=F(t)$, the production function is expressed as where $\mathscr{M}(t)=x(t) F(t)=x(t) D(t)$ is a continuous function denoting the production of unit time of the microorganism. Since we will focus on the maximum microorganism production problem in the interval $[0, T]$, the objective functional is given as

$$
J(D(\cdot))=\mathbb{E}\{M(T)\}=\mathbb{E}\left\{\int_{0}^{T} x(t) D(t) \mathrm{d} t\right\} .
$$

Denote a bounded nonempty closed set $\mathscr{A} \subset \mathbb{R}$, and the set of admissible controls is defined as follows:

$$
\mathscr{A}_{a d} \triangleq\left\{D:[0, T] \times \Omega \longrightarrow \mathscr{A} \mid D \text { is }\left\{\mathscr{F}_{t}\right\}_{t \geq 0} \text { - adapted }\right\} .
$$

For any $D \in \mathscr{A}_{a d}$, equation (87) is a stochastic differential equation with random coefficients, which has a unique strong $(S(t), x(t), D(t))$ is called an admissible triple.

The objective functional (90) can be rewritten as

$$
\begin{aligned}
J(D(\cdot)) \triangleq & \mathbb{E}\left\{\int_{0}^{T} L(S(t), x(t), D(t)) \mathrm{d} t\right\}, \\
& \mathbb{E}\left\{\int_{0}^{T}|L(S(t), x(t), D(t))| \mathrm{d} t\right\}<\infty,
\end{aligned}
$$

where $L(S(t), x(t), D(t))=x(t) D(t)$, the optimal control condition is to select a nonanticipative decision that maximize the objective functional, that is to seek $\widehat{D} \in \mathscr{A}_{a d}$ such that

$$
J(\widehat{D})=\sup _{D \in \mathscr{A}_{a d}} J(D),
$$

where $\widehat{D}$ is called an optimal stochastic control. $(\widehat{S}, \widehat{x})$ is called the corresponding solution of the model (87) and $(\widehat{S}, \widehat{x}, \widehat{D})$ is called an optimal triple.

Define a Hamiltonian function $H: \mathbb{R}_{+} \times \mathbb{R}_{+} \times \mathscr{A} \times \mathbb{R} \times$ $\mathbb{R} \times \mathbb{R} \times \mathbb{R} \longrightarrow \mathbb{R}$ by

$$
\begin{aligned}
& H\left(S(t), x(t), D(t), p_{1}(t), p_{2}(t), q_{1}(t), q_{2}(t)\right) \\
&= f_{1}(S(t), x(t), D(t)) p_{1}(t)+f_{2}(S(t), x(t), D(t)) p_{2}(t) \\
&+\sigma_{1} S(t) q_{1}(t)+\sigma_{2} x(t) q_{2}(t) \\
&+L(S(t), x(t), D(t)) .
\end{aligned}
$$

The adjoint equation corresponding to the admissible triple $(S(t), x(t), D(t))$ on the unknown $\left\{\mathscr{F}_{t}\right\}_{t \geq 0}$-adapted processes $\left(p_{1}(t), p_{2}(t), q_{1}(t), q_{2}(t)\right)$ is the backward stochastic differential equation with the natural boundary conditions: solution $(S(t), x(t))$ called an admissible state trajectory, and 


$$
\left\{\begin{array}{l}
\mathrm{d} p_{1}(t)=-g_{1}\left(S(t), x(t), D(t), p_{1}(t), p_{2}(t), q_{1}(t), q_{2}(t)\right) \mathrm{d} t+q_{1}(t) \mathrm{d} B_{1}(t) \\
\mathrm{d} p_{2}(t)=-g_{2}\left(S(t), x(t), D(t), p_{1}(t), p_{2}(t), q_{1}(t), q_{2}(t)\right) \mathrm{d} t+q_{2}(t) \mathrm{d} B_{2}(t) \\
p_{1}(T)=0 \\
p_{2}(T)=0
\end{array}\right.
$$

where

$$
\begin{aligned}
g_{1}\left(S, x, D, p_{1}, p_{2}, q_{1}, q_{2}\right)= & -\left(D+\frac{m x\left(A k x-B S^{2}\right)}{(k x+S)^{2}(A+B S)^{2}}\right) p_{1} \\
& +\frac{m k x^{2}}{(k x+S)^{2}} p_{2}+\sigma_{1} q_{1}, \\
g_{2}\left(S, x, D, p_{1}, p_{2}, q_{1}, q_{2}\right)= & -\frac{m S^{2}}{(k x+S)^{2}(A+B S)} p_{1} \\
& +\left(\frac{m S^{2}}{(k x+S)^{2}}-D\right) p_{2}+\sigma_{2} q_{2}+D .
\end{aligned}
$$

Next, the necessary condition of the optimal stochastic control for the model (4) is established.

Theorem 5. Suppose that there exists an adapted solution $\left(\widehat{p}_{1}, \widehat{p}_{2}, \widehat{q}_{1}, \widehat{q}_{2}\right)$ of the corresponding adjoint equation (95) such that for all $D \in \mathscr{A}_{a}$, we have

$$
\mathbb{E}\left\{\int_{0}^{T}\left[(\widehat{S}-S)^{2} \widehat{q}_{1}^{2}+(\widehat{x}-x)^{2} \widehat{q}_{2}^{2}\right] \mathrm{d} t\right\}<\infty,
$$

and

$$
\mathbb{E}\left\{\int_{0}^{T}\left[\sigma_{1}^{2} S^{2} \widehat{p}_{1}^{2}+\sigma_{2}^{2} x^{2} \widehat{p}_{2}^{2}\right] \mathrm{d} t\right\}<\infty .
$$

Furthermore, if the following conditions hold,

(i) The Hamiltonian $H\left(S, x, D, \widehat{p}_{1}, \widehat{p}_{2}, \widehat{q}_{1}, \widehat{q}_{2}\right)$ with respect to $(S, x, D)$ is concave for all $t \in[0, T]$

(ii) $H\left(\widehat{S}, \widehat{x}, \widehat{D}, \widehat{p}_{1}, \widehat{p}_{2}, \widehat{q}_{1}, \widehat{q}_{2}\right)=$

$\max _{D \in \mathscr{A}} H\left(\widehat{S}, \widehat{x}, D, \widehat{p}_{1}, \widehat{p}_{2}, \widehat{q}_{1}, \widehat{q}_{2}\right), \quad$ for all $t \in[0, T]$

then, $(\widehat{S}, \widehat{x}, \widehat{D})$ is an optimal triple and $\widehat{D}$ is an optimal stochastic control.

Proof. Proof. For any control triple $(S, x, D) \in \mathscr{A}_{a d}$, we consider

$$
\begin{aligned}
J(\widehat{D})-J(D) & =\mathbb{E}\left\{\int_{0}^{T} L(\widehat{S}, \widehat{x}, \widehat{D}) \mathrm{d} t\right\}-\mathbb{E}\left\{\int_{0}^{T} L(S, x, D) \mathrm{d} t\right\} \\
& =\mathbb{E}\left\{\int_{0}^{T}[L(\widehat{S}, \widehat{x}, \widehat{D})-L(S, x, D)] \mathrm{d} t\right\} \triangleq I .
\end{aligned}
$$

By the Hamiltonian function $H$ and the assumption of $\left(\widehat{p}_{1}, \widehat{p}_{2}, \widehat{q}_{1}, \widehat{q}_{2}\right)$, we have

$$
\begin{aligned}
L(S, x, D)= & H\left(S, x, D, \widehat{p}_{1}, \widehat{p}_{2}, \widehat{q}_{1}, \widehat{q}_{2}\right)-f_{1}(S, x, D) \widehat{p}_{1} \\
& -f_{2}(S, x, D) \widehat{p}_{2}-\sigma_{1} S \widehat{q}_{1}-\sigma_{2} x \widehat{q}_{2},
\end{aligned}
$$

therefore

$$
\begin{aligned}
I= & \mathbb{E}\left\{\int_{0}^{T}\left[H\left(\widehat{S}, \widehat{x}, \widehat{D}, \widehat{p}_{1}, \widehat{p}_{2}, \widehat{q}_{1}, \widehat{q}_{2}\right)-H\left(S, x, D, \widehat{p}_{1}, \widehat{p}_{2}, \widehat{q}_{1}, \widehat{q}_{2}\right)\right] \mathrm{d} t\right\} \\
& -\mathbb{E}\left\{\int_{0}^{T}\left[f_{1}(\widehat{S}, \widehat{x}, \widehat{D}) \widehat{p}_{1}-f(S, x, D) \widehat{p}_{1}\right] \mathrm{d} t\right\}-\mathbb{E}\left\{\int_{0}^{T}\left[f_{2}(\widehat{S}, \widehat{x}, \widehat{D}) \widehat{p}_{2}-f_{2}(S, x, D) \widehat{p}_{2}\right] \mathrm{d} t\right\} \\
& -\mathbb{E}\left\{\int_{0}^{T}\left[\sigma_{1} \widehat{S}_{\hat{q}_{1}}-\sigma_{1} S \widehat{q}_{1}\right] \mathrm{d} t\right\}-\mathbb{E}\left\{\int_{0}^{T}\left[\sigma_{2} \widehat{x} \widehat{q}_{2}-\sigma_{2} x \widehat{q}_{2}\right] \mathrm{d} t\right\} .
\end{aligned}
$$

Since the concavity of $H$ in $S, x$ and $D$, we get

$$
\begin{aligned}
I \geq & \mathbb{E}\left\{\int_{0}^{T}\left[(\widehat{S}-S) H_{S}\left(\widehat{S}, \widehat{x}, \widehat{D}, \widehat{p}_{1}, \widehat{p}_{2}, \widehat{q}_{1}, \widehat{q}_{2}\right)+(\widehat{x}-x) H_{x}\left(\widehat{S}, \widehat{x}, \widehat{D}, \widehat{p}_{1}, \widehat{p}_{2}, \widehat{q}_{1}, \widehat{q}_{2}\right)\right] \mathrm{d} t\right\} \\
& +\mathbb{E}\left\{\int_{0}^{T}\left[(\widehat{D}-D) H_{D}\left(\widehat{S}, \widehat{x}, \widehat{D}, \widehat{p}_{1}, \widehat{p}_{2}, \widehat{q}_{1}, \widehat{q}_{2}\right)\right] \mathrm{d} t\right\}-\mathbb{E}\left\{\int_{0}^{T}\left[f_{1}(\widehat{S}, \widehat{x}, \widehat{D}) \widehat{p}_{1}-f_{1}(S, x, D) \widehat{p}_{1}\right] \mathrm{d} t\right\} \\
& -\mathbb{E}\left\{\int_{0}^{T}\left[f_{2}(\widehat{S}, \widehat{x}, \widehat{D}) \widehat{p}_{2}-f_{2}(S, x, D) \widehat{p}_{2}\right] \mathrm{d} t\right\}-\mathbb{E}\left\{\int_{0}^{T}\left[\sigma_{1} \widehat{S}_{\hat{q}_{1}}-\sigma_{1} S \widehat{q}_{1}\right] \mathrm{d} t\right\} \\
& -\mathbb{E}\left\{\int_{0}^{T}\left[\sigma_{2} \hat{x} \widehat{q}_{2}-\sigma_{2} x \widehat{q}_{2}\right] \mathrm{d} t\right\} \triangleq I_{1}+I_{2},
\end{aligned}
$$


where

$$
\begin{aligned}
& I_{1}=\mathbb{E}\left\{\int_{0}^{T}\left[(\widehat{S}-S) H_{S}\left(\widehat{S}, \widehat{x}, \widehat{D}, \widehat{p}_{1}, \widehat{p}_{2}, \widehat{q}_{1}, \widehat{q}_{2}\right)+(\widehat{x}-x) H_{x}\left(\widehat{S}, \widehat{x}, \widehat{D}, \widehat{p}_{1}, \widehat{p}_{2}, \widehat{q}_{1}, \widehat{q}_{2}\right)\right] \mathrm{d} t\right\} \\
& -\mathbb{E}\left\{\int_{0}^{T}\left[f_{1}(\widehat{S}, \widehat{x}, \widehat{D}) \widehat{p}_{1}-f_{1}(S, x, D) \widehat{p}_{1}\right] \mathrm{d} t\right\}-\mathbb{E}\left\{\int_{0}^{T}\left[f_{2}(\widehat{S}, \widehat{x}, \widehat{D}) \widehat{p}_{2}-f_{2}(S, x, D) \hat{p}_{2}\right] \mathrm{d} t\right\} \\
& -\mathbb{E}\left\{\int_{0}^{T}\left[\sigma_{1} \hat{S} \widehat{q}_{1}-\sigma_{1} S \widehat{q}_{1}\right] \mathrm{d} t\right\}-\mathbb{E}\left\{\int_{0}^{T}\left[\sigma_{2} \widehat{x} \widehat{q}_{2}-\sigma_{2} x \widehat{q}_{2}\right] \mathrm{d} t\right\}, \\
& I_{2}=\mathbb{E}\left\{\int_{0}^{T}\left[(\widehat{D}-D) H_{D}\left(\widehat{S}, \widehat{x}, \widehat{D}, \hat{p}_{1}, \widehat{p}_{2}, \widehat{q}_{1}, \widehat{q}_{2}\right)\right] \mathrm{d} t\right\} \text {. }
\end{aligned}
$$

Conditions (97) and (98) ensure that the stochastic integrals have zero mean. Next, we will estimate $I_{1}$ by Itô product formula and the natural boundary conditions of (95).

$$
\begin{aligned}
I_{1}= & \mathbb{E}\left\{\int_{0}^{T}\left[(\widehat{S}-S) H_{S}\left(\widehat{S}, \widehat{x}, \widehat{D}, \widehat{p}_{1}, \widehat{p}_{2}, \widehat{q}_{1}, \widehat{q}_{2}\right)-\left(f_{1}(\widehat{S}, \widehat{x}, \widehat{D})-f_{1}(S, x, D)\right) \widehat{p}_{1}-(\widehat{S}-S) \sigma_{1} \widehat{q}_{1}\right] \mathrm{d} t\right\} \\
& +\mathbb{E}\left\{\int_{0}^{T}\left[(\widehat{x}-x) H_{x}\left(\widehat{S}, \widehat{x}, \widehat{D}, \widehat{p}_{1}, \widehat{p}_{2}, \widehat{q}_{1}, \widehat{q}_{2}\right)-\left(f_{2}(\widehat{S}, \widehat{x}, \widehat{D})-f_{2}(S, x, D)\right) \widehat{p}_{2}-(\widehat{x}-x) \sigma_{2} \widehat{q}_{2}\right] \mathrm{d} t\right\} \\
= & -\mathbb{E}\left\{\int_{0}^{T}(\widehat{S}-S) \mathrm{d} \widehat{p}_{1}+\int_{0}^{T} \widehat{p}_{1} \mathrm{~d}(\widehat{S}-S)+\int_{0}^{T} \mathrm{~d} \widehat{p}_{1} \mathrm{~d}(\widehat{S}-S)\right\} \\
& -\mathbb{E}\left\{\int_{0}^{T}(\widehat{x}-x) \mathrm{d} \widehat{p}_{2}+\int_{0}^{T} \widehat{p}_{2} \mathrm{~d}(\widehat{x}-x)+\int_{0}^{T} \mathrm{~d} \widehat{p}_{2} \mathrm{~d}(\widehat{x}-x)\right\} \\
= & -\mathbb{E}\left\{(\widehat{S}(T)-S(T)) \widehat{p}_{1}(T)\right\}-\mathbb{E}\left\{(\widehat{x}(T)-x(T)) \widehat{p}_{2}(T)\right\} \\
= & 0 .
\end{aligned}
$$

Applying the condition (ii) of theorem,

$$
\begin{aligned}
& \mathbb{E}\left\{(\widehat{D}(t)-D(t)) H_{D}\left(\widehat{S}, \widehat{x}, \widehat{D}, \widehat{p}_{1}, \widehat{p}_{2}, \widehat{q}_{1}, \widehat{q}_{2}\right)\right\} \\
= & (\widehat{D}(t)-D(t)) \mathbb{E}\left\{H_{D}\left(\widehat{S}, \widehat{x}, D, \widehat{p}_{1}, \widehat{p}_{2}, \widehat{q}_{1}, \widehat{q}_{2}\right)\right\}_{D=\widehat{D}} \geq 0,
\end{aligned}
$$

then

$$
I_{2}=\mathbb{E}\left\{\int_{0}^{T}\left[(\widehat{D}-D) H_{D}\left(\widehat{\mathcal{S}}, \widehat{x}, \widehat{D}, \widehat{p}_{1}, \widehat{p}_{2}, \widehat{q}_{1}, \widehat{q}_{2}\right)\right] \mathrm{d} t\right\} \geq 0 .
$$

Hence, we have $I=I_{1}+I_{2} \geq 0$; that is,

$$
J(\widehat{D}) \geq J(D) \text {. }
$$

Considering $(S, x, D)$ is arbitrary, then $(\widehat{S}, \widehat{x}, \widehat{D})$ is an optimal triple and $\widehat{D}$ is an optimal stochastic control. This completes the proof of Theorem 5 .

Since the control variable $D$ appears linearly in the Hamiltonian function $H\left(S, x, D, p_{1}, p_{2}, q_{1}, q_{2}\right)$. This indicates that the problem may have an optimal stochastic singular control. However, it is usually difficult to find the exact optimal stochastic singular control. To this end, we will introduce a small perturbation to turn the singular control into a nonsingular control and call it a near-optimal stochastic control of this problem.

Let the objective functional is

$$
J^{*}(D(\cdot))=\mathbb{E}\left\{\int_{0}^{T}\left(x(t) D(t)-\frac{1}{2} \varepsilon D(t)^{2}\right) \mathrm{d} t\right\} .
$$

where $(1 / 2) \varepsilon D(t)^{2}$ represents a small perturbation, and the positive constant $\varepsilon$ is small enough to ensure $x(t) D(t)-(1 / 2) \varepsilon D(t)^{2}>0$. Thus, the Hamiltonian function can be rewritten as

$$
\begin{aligned}
H^{*} & \left(S(t), x(t), D(t), p_{1}(t), p_{2}(t), q_{1}(t), q_{2}(t)\right) \\
\quad & =f_{1}(S(t), x(t), D(t)) p_{1}(t)+f_{2}(S(t), x(t), D(t)) p_{2}(t) \\
& +\sigma_{1} S(t) q_{1}(t)+\sigma_{2} x(t) q_{2}(t)+x(t) D(t)-\frac{1}{2} \varepsilon D(t)^{2} .
\end{aligned}
$$

The necessary conditions for the near-optimal stochastic control are $H_{D}^{*}=0$ and $H_{D D}^{*}<0$ at the point $\left(\widehat{S}, \widehat{x}, \widehat{D}, \widehat{p_{1}}, \widehat{p}_{2}, \widehat{q_{1}}, \widehat{q_{2}}\right)$. Hence, we get the following result. 
Corollary 1. The model (4) has a near-optimal stochastic control:

$$
\widehat{D}=\frac{1}{\varepsilon}\left(\left(S^{0}-\widehat{S}\right) \widehat{p}_{1}-\widehat{x} \widehat{p}_{2}+\widehat{x}\right),
$$

and the corresponding optimal triple $(\widehat{S}, \widehat{x}, \widehat{D})$.

\section{Numerical Simulations}

Numerical simulations are presented for supporting our theoretical results of the model (4) by Milstein method [33]. The discretized equations are listed as follows:

$$
\left\{\begin{array}{l}
S_{i+1}=S_{i}+\left(\left(S^{0}-S_{i}\right) D-\frac{m S_{i}}{k x_{i}+S_{i}} \frac{x_{i}}{A+B S_{i}}\right) \Delta t+S_{i}\left(\sigma_{1} \xi_{i} \sqrt{\Delta t}+\frac{\sigma_{1}^{2}}{2}\left(\xi_{i}^{2}-1\right) \Delta t\right), \\
x_{i+1}=x_{i}+x_{i}\left(\frac{m S_{i}}{k x_{i}+S_{i}}-D\right) \Delta t+x_{i}\left(\sigma_{2} \zeta_{i} \sqrt{\Delta t}+\frac{\sigma_{2}^{2}}{2}\left(\zeta_{i}^{2}-1\right) \Delta t\right),
\end{array}\right.
$$

where $\xi_{i}, \zeta_{i},(i=1,2, \ldots)$ are independent $\mathbb{N}(0,1)$-distributed Gaussian random variables.

Next, we present numerical simulations to demonstrate the results.

Example 1. For the model (3) and (4), choose two sets of parameters:

$S^{0}=2, m=0.5, k=1, D=1, A=2, B=0, \sigma_{1}=$

$0.1, \sigma_{2}=1.2$, Figures $1(\mathrm{a})$ and $1(\mathrm{~b})$

and

$S^{0}=2, m=0.5, k=1, D=1, A=2, B=3, \sigma_{1}=$

$0.1, \sigma_{2}=1.2$, Figures $1(\mathrm{c})$ and $1(\mathrm{~d})$

and the initial point is $\left(S_{0}, x_{0}\right)=(0.4,0.5)$. Obviously,

$$
m<D \text {, }
$$

which means that when the microorganism is extinct without reference to the intensity of noise (see Figure 1).

Example 2. For the model (3) and (4), choose two sets of parameters:

$S^{0}=2, m=1.45, k=1, D=1, A=2, B=0, \sigma_{1}=$ $0.1, \sigma_{2}=1.2$, Figures $2(\mathrm{a})$ and $2(\mathrm{~b})$

and

$S^{0}=2, m=1.45, k=1, D=1, A=2, B=3, \sigma_{1}=$ $0.1, \sigma_{2}=1.2$, Figures $2(\mathrm{c})$ and $2(\mathrm{~d})$

and the initial point is $\left(S_{0}, x_{0}\right)=(0.4,0.5)$. Since

$$
\begin{aligned}
\sigma_{2}^{2} & =1.44, \\
2(m-D) & =0.9,
\end{aligned}
$$

obviously,

$$
\sigma_{2}^{2}>2(m-D)
$$

which means that when the condition of Theorem 2 satisfies, then the microorganism is extinct, though the microorganism $x(t)$ in deterministic model (3) is persistent (see Figure 2).

Example 3. For the model (3) and (4), choose two sets of parameters:
$S^{0}=1.1, m=1.4, k=1, D=0.1, H_{2}=10, A=2, B=0$, $\sigma_{1}=0.15, \sigma_{2}=0.15$, Figures 3(a) and 3(b)

and

$S^{0}=1.1, m=1.4, k=1, D=0.1, H_{2}=10, A=2, B=3$, $\sigma_{1}=0.15, \sigma_{2}=0.15$, Figures $3(\mathrm{c})$ and $3(\mathrm{~d})$

and the initial point is $\left(S_{0}, x_{0}\right)=(0.4,0.5)$. Since

$$
\begin{aligned}
\frac{1}{2} \sigma_{2}^{2} & \approx 0.0113, \\
\frac{m}{k H_{2}+1}-D & \approx 0.0273, \\
\frac{m S^{0}}{k H_{2}+1}-D & =0.0400,
\end{aligned}
$$

obviously,

$$
\frac{1}{2} \sigma_{2}^{2}<\frac{m}{k H_{2}+1}-D<\frac{m S^{0}}{k H_{2}+1}-D,
$$

which means that when the condition of Theorem 3 satisfies, then the microorganism is almost surely strongly persistent in the mean (see Figure 3).

Example 4. For the model (3) and (4), choose two sets of parameters:

$$
\begin{aligned}
& S^{0}=0.9, m=1.4, k=1, D=0.1, H_{2}=10, A=2, B=0, \\
& \sigma_{1}=0.15, \sigma_{2}=0.15, \text { Figures } 4(\mathrm{a}) \text { and } 4(\mathrm{~b}) \\
& \text { and } \\
& S^{0}=0.9, m=1.4, k=1, D=0.1, H_{2}=10, A=2, B=3, \\
& \sigma_{1}=0.15, \sigma_{2}=0.15, \text { Figures } 4(\mathrm{c}) \text { and } 4(\mathrm{~d})
\end{aligned}
$$

and the initial point is $\left(S_{0}, x_{0}\right)=(0.4,0.5)$. Since

$$
\begin{aligned}
\frac{1}{2} \sigma_{2}^{2} & \approx 0.0113, \\
\frac{m S^{0}}{k H_{2}+1}-D & \approx 0.0145, \\
\frac{m}{k H_{2}+1}-D & \approx 0.0273,
\end{aligned}
$$


obviously,

$$
\frac{1}{2} \sigma_{2}^{2}<\frac{m S^{0}}{k H_{2}+1}-D<\frac{m}{k H_{2}+1}-D,
$$

which means that when the condition of Theorem 3 satisfies, then the microorganism is almost surely strongly persistent in the mean (see Figure 4).

Through the above numerical simulations, we know that the parameters $B$ rarely affect the extinction of the microorganism $x(t)$ and have a significant impact on the persistence of the microorganism $x(t)$, that is, the concentration of the microorganism $x(t)$ increases with the parameter $B$ (see Figures 3(b), 3(d), 4(b), and 4(d)). In conclusion, the intensities of white noise are disadvantageous to the growth of the microorganism, and the variable yield $\gamma(S)=A+B S$ is advantageous to the growth of the microorganism.

\section{Data Availability}

The "simulation" data used to support the findings of this study are included within the article. These data are not obtained by experiments; just to satisfy the conditions of the theorem, we design them artificially.

\section{Conflicts of Interest}

The authors declare that they have no conflicts of interest.

\section{Acknowledgments}

The work was supported by the Natural Science Foundation of Shanxi Province of China (201801D121011).

\section{References}

[1] H. L. Smith and P. Waltman, The Theory of the Chemostat: Dynamics of Microbial Competition, Cambridge University Press, Cambridge, UK, 1995.

[2] S. L. Sun and H. Yin, "Qualitative analysis of a predator-prey model with different delays in the chemostat model," Journal of System Science and Mathematical Sciences, vol. 36, pp. 2454-2472, 2016, in Chinese.

[3] L. Wang, D. Jiang, and D. O'Regan, "The periodic solutions of a stochastic chemostat model with periodic washout rate," Communications in Nonlinear Science and Numerical Simulation, vol. 37, pp. 1-13, 2016.

[4] T. Bayen, A. Rapaport, and F.-Z. Tani, "Optimal periodic control of the chemostat with Contois growth function," IFAC-PapersOnLine, vol. 51, no. 2, pp. 730-734, 2018.

[5] S. L. Sun and L. S. Chen, "Asymptotic behavior of ratiodependent chemostat model with varibale yield," Journal of Dalian University of Technology, vol. 47, pp. 931-936, 2007, in Chinese.

[6] A. G. Dorofeev, M. V. Glagolev, T. F. Bondarenko, and N. S. Panikov, "Observation and explanation of the unusual growth kinetics of Arthrobacter globiforms," Microbiology, vol. 61, pp. 33-42, 1992.

[7] Z. Li, L. Chen, and Z. Liu, "Periodic solution of a chemostat model with variable yield and impulsive state feedback control," Applied Mathematical Modelling, vol. 36, no. 3, pp. 1255-1266, 2012.
[8] X. Huang, L. Zhu, and E. H. C. Chang, "Limit cycles in a chemostat with general variable yields and growth rates," Nonlinear Analysis: Real World Applications, vol. 8, no. 1, pp. 165-173, 2007.

[9] G. Fu and W. Ma, "Hopf bifurcations of a variable yield chemostat model with inhibitory exponential substrate uptake," Chaos, Solitons \& Fractals, vol. 30, no. 4, pp. 845-850, 2006.

[10] R. Yan and S. Sun, "Stochastic characteristics of a chemostat model with variable yield," Physica A: Statistical Mechanics and Its Applications, vol. 537, Article ID 122681, 2020.

[11] L. Imhof and S. Walcher, "Exclusion and persistence in deterministic and stochastic chemostat models," Journal of Differential Equations, vol. 217, no. 1, pp. 26-53, 2005.

[12] X. Zhang and R. Yuan, "The existence of stationary distribution of a stochastic delayed chemostat model," Applied Mathematics Letters, vol. 93, pp. 15-21, 2019.

[13] S. Sun, Y. Sun, G. Zhang, and X. Liu, "Dynamical behavior of a stochastic two-species Monod competition chemostat model," Applied Mathematics and Computation, vol. 298, pp. 153-170, 2017.

[14] S. L. Sun and X. F. Zhang, "Asymptotic behavior of a stochastic delayed chemostat model with nutrient storange," Journal of Biological Systems, vol. 25, pp. 1-22, 2018.

[15] X. Mao, "Stationary distribution of stochastic population systems," Systems \& Control Letters, vol. 60, no. 6, pp. 398-405, 2011.

[16] Q. Liu, D. Jiang, T. Hayat, and A. Alsaedi, "Stationary distribution and extinction of a stochastic HIV-1 model with Beddington-DeAngelis infection rate," Physica A: Statistical Mechanics and Its Applications, vol. 512, pp. 414-426, 2018.

[17] M. Liu, K. Wang, and Q. Wu, "Survival analysis of stochastic competitive models in a polluted environment and stochastic competitive exclusion principle," Bulletin of Mathematical Biology, vol. 73, no. 9, pp. 1969-2012, 2011.

[18] C. Ji and D. Jiang, "Threshold behaviour of a stochastic SIR model," Applied Mathematical Modelling, vol. 38, no. 21-22, pp. 5067-5079, 2014.

[19] N. Dalal, D. Greenhalgh, and X. Mao, "A stochastic model for internal HIV dynamics," Journal of Mathematical Analysis and Applications, vol. 341, no. 2, pp. 1084-1101, 2008.

[20] G. Stephanopoulos, R. Aris, and A. G. Fredrickson, "A stochastic analysis of the growth of competing microbial populations in a continuous biochemical reactor," Mathematical Biosciences, vol. 45, no. 1-2, pp. 99-135, 1979.

[21] J. R. Beddington and R. M. May, "Harvesting natural populations in a randomly fluctuating environment," Science, vol. 197, no. 4302, pp. 463-465, 1977.

[22] A. Haddon and C. Hermosilla, "An algorithm for maximizing the biogas production in a chemostat," Journal of Optimization Theory and Applications, vol. 182, no. 3, pp. 1150-1170, 2019.

[23] D. Ding, Z. Wang, H. Dong, and H. Shu, "Distributed $H_{\infty}$ state estimation with stochastic parameters and nonlinearities through sensor networks: the finite-horizon case," Automatica, vol. 48, no. 8, pp. 1575-1585, 2012.

[24] W. Guo, Q. Zhang, and L. Rong, "A stochastic epidemic model with nonmonotone incidence rate: sufficient and necessary conditions for near-optimality," Information Sciences, vol. 467, pp. 670-684, 2018.

[25] N. C. Framstad, B. Øksendal, A. Sulem, and F. Zirilli, "Sufficient stochastic maximum principle for the optimal control of jump diffusions and applications to finance," Journal of 
Optimization Theory and Applications, vol. 121, no. 1, pp. 77-98, 2004.

[26] L. Chen and J. Huang, "Stochastic maximum principle for controlled backward delayed system via advanced stochastic differential equation," Journal of Optimization Theory and Applications, vol. 167, no. 3, pp. 1112-1135, 2015.

[27] J. Yong and X. Y. Zhou, Stochastic Controls Hamiltonian Systems and HJB Equations, Springer-Verlag, New York, NY, USA, 1999.

[28] X. R. Mao, Stochastic Differential Equations and Applications, Horwood Publishing, Chichester UK, 2nd edition, 2007.

[29] H. H. Kuo, Introduction to Stochstic Integration, Birkhauser, Basel, Switzerland, 1983.

[30] R. Khasminskii, Stochastic Stability of Differential Equations, Sijthoff and Noordhoff, Amsterdam, Netherlands, 1980.

[31] T. C. Gard, Introduction to Stochastic Differential Equations, Marcel Dekker, New York, NY, USA, 1988.

[32] G. Strang, Linear Algebra and Its Applications, Thomson Learning INC, London, UK, 1988.

[33] D. J. Higham, "An algorithmic introduction to numerical simulation of stochastic differential equations," SIAM Review, vol. 43, no. 3, pp. 525-546, 2001. 